UNIVERSITE் DU QUÉBEC

\author{
MEMMOIRE \\ PRÉSENTÉ A \\ I'UNIVERSITÉ DU QUÉBEC A CHICOUTIMI \\ COMME EXIGENCE PARTIELLE \\ DE LA MAITRISE EN GESTION DES PMO
}

\author{
PAR \\ JEAN-PIERRE LACHANCE \\ B.sc.soc. \\ LES CONDITIONS DE SUCCES DES PME \\ INTÉGRANT DE NOUVELLES TECHNOLOGIES: \\ LES ASPECTS ASSOCIÉS AUX \\ ACTIVITÉS DE FORMATION
}

JANVIER 1994 


\section{Bibliothèque}

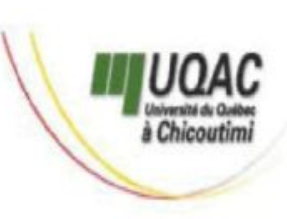

\section{Mise en garde/Advice}

Afin de rendre accessible au plus Motivated by a desire to make the grand nombre le résultat des results of its graduate students' travaux de recherche menés par ses research accessible to all, and in étudiants gradués et dans l'esprit des accordance with the rules règles qui régissent le dépôt et la governing the acceptation and diffusion des mémoires et thèses diffusion of dissertations and produits dans cette Institution, theses in this Institution, the I'Université du Québec à Université du Québec à Chicoutimi (UQAC) est fière de Chicoutimi (UQAC) is proud to rendre accessible une version make a complete version of this complète et gratuite de cette œuvre. work available at no cost to the reader.

L'auteur conserve néanmoins la The author retains ownership of the propriété du droit d'auteur qui copyright of this dissertation or protège ce mémoire ou cette thèse. thesis. Neither the dissertation or Ni le mémoire ou la thèse ni des thesis, nor substantial extracts from extraits substantiels de ceux-ci ne it, may be printed or otherwise peuvent être imprimés ou autrement reproduced without the author's reproduits sans son autorisation. permission. 


\section{RËSUME்}

Devant une concurrence qui devient de plus en plus vive, les organisations n'ont guère le choix que de recourir à de nouvelles technologies pour demeurer compétitives. Face à des problèmes de productivité, les grandes entreprises ont pris les moyens pour augmenter leur taux de diffusion de nouvelles technologies. Le passage des PME vers l'usine du futur n'est pas sans embûches. L'adoption de nouvelles technologies ne se limite pas à l'acquisition de l'équipement mais a aussi des répercussions sur la dimension humaine des organisations.

Selon une étude américaine, 50 à 75 pour cent des entreprises qui ont fait l'adoption de nouvelles machineries reliées à la productique ont connu des échecs causés par des problèmes humains ou organisationnels. Parmi les facteurs de reussites, le style de gestion, la mise en valeur des ressources humaines, le recyclage, la formation continue, et un climat de travail positif sont souvent mentionnés.

A partir d'un modèle conceptuel développé par Ann Majchrzak servant à analyser l'infrastructure humaine des organisations avant l'achat d'équipement pour déterminer les impacts sur le choix de la machinerie et la dimension humaine, nous avons appliqué celui-ci à des PME oeuvrant dans des ateliers d'usinage au Saguenay Lac st-Jean en s'intéressant particulièrement aux activités de formation. Selon les dirigeants rencontrés, le but visé par l'adoption de nouvelles technologies était relié à l'augmentation de la productivité ou l'amélioration de la qualité. Par ailleurs, certains ont mis en place des mécanismes de consultation auprès du personnel pour les impliquer dans l'adoption des nouveaux équipements alors que pour d'autres, la décision leur appartenait seul. Certains gestionnaires, se butent à de la résistance de leur personnel alors que d'autres profitent d'un climat de travail favorable.

En conclusion, les entrepreneurs mentionnent que la sélection de l'équipement, la présence dans le marché, l'implication du personnel et les activités de formation sont les variables par ordre d'importance représentant la clé du succès dans l'adoption d'un programme de productique. 


\section{AVANT-PROPOS}

Oeuvrant à titre d'économiste régional pour le Ministère d'Emploi et Immigration Canada au Saguenay Lac St-Jean, le sujet de la formation en main-d'oeuvre constitue un des éléments de nos analyses et recherches. Ce thème prend une place de plus en plus prépondérante dans l'actualité de tous les jours et pourrait constituer le défi des années 1990. Par ailleurs, les écrits et recherches sur ce sujet et s'adressant plus particulièrement aux petites et moyennes organisations demeurent peu nombreuses.

J'espère que ce mémoire permettra de lever une partie du voile qui recouvre encore ce champ d'études. Bien que compte tenu du nombre limité d'entreprises sous étude, les résultats ne permettent pas de généraliser, il est à souhaiter que ceux-ci puissent servir de points de départs à d'autres travaux ou recherches sur le thème de la formation de la main-d'oeuvre.

Je profite de l'occasion pour remercier mon directeur de mémoire Monsieur Gilles St-Pierre pour ses conseils et son 
support ainsi que Madame Doris Tremblay qui a agit à titre de lectrice et de correctrice en plus d'assurer le traitement informatique de ce document. 
TABLE DES MATIERES

RESUME..............................

AVANT-PROPOS.............................. . . .

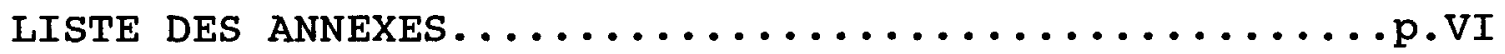

INTRODUCTION.......................... 1

CHAPITRE I

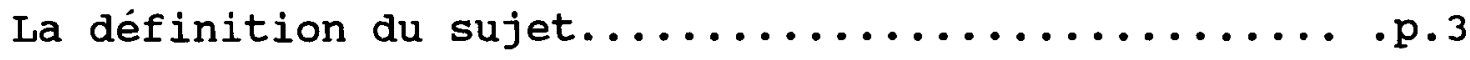

CHAPITRE II

Problèmatique............................ p.10

CHAPITRE III

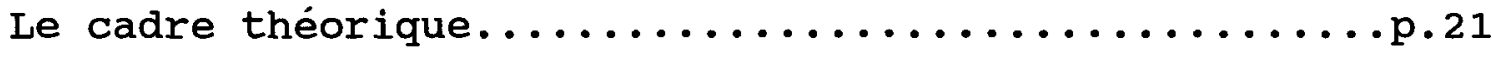

CHAPITRE IV

Cadre méthodologique........................... 40 
CHAPITRE V

Etudes de cas...............................46

Entreprise A............................. p.46

Entreprise B................................. 53

Entreprise c................................ .59

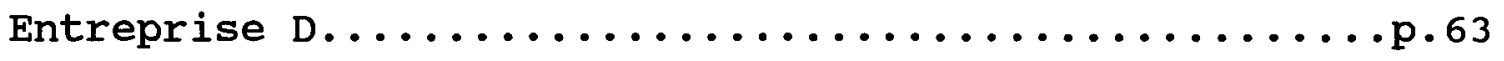

CHAPITRE VI

Analyse des résultats......................... 69

CONCLUSION.............................. 80

BIBLIOGRAPHIE............................ 84 


\title{
LISTE DES ANNEXES
}

\begin{abstract}
ANNEXE I.
Description des nouvelles technologies selon le niveau de

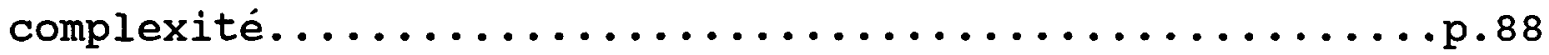

\begin{abstract}
ANNEXE 11.
Etapes à suivre pour l'identification des impacts reliés aux ressources humaines suite à l'adoption de nouvelles

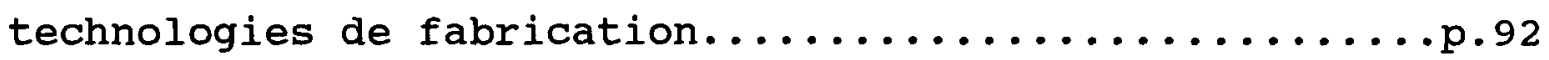

ANNEXE III.

Guide d'entrevue..............................93 
INTRODUCTION

Le Québec et le Canada ne sont guère réputés pour le montant investi par entreprise dans la formation de leur personnel. L'organisation de coopération et de développement èconomique (OCDE) a classé le Canada au 16 ième rang de ses pays membres, en 1990, selon les dépenses consacrées à la formation en proportion du produit intérieur brut.

Compte tenu que devant la nécessité de diminuer les coûts de production ou améliorer leur productivité, les entreprises tendent de plus en plus à prendre le virage technologique. Cependant, l'adoption de nouvelles technologies ne se limite pas à l'investissement monétaire en machinerie, mais comporte également des effets sur l'organisation humaine des entreprises dont en particulier, sur les activités de formation.

Ainsi, à l'aide d'études de cas menées auprès d'entreprises du secteur manufacturier qui ont fait l'acquisition de nouvelles technologies, nous tenterons de faire ressortir les conditions de succès reliées aux 
activités de formation. Il faut mentionner que selon des études américaines sur le sujet, suite à l'introduction d'un programme de productique, les gestionnaires ont fait face à des problèmes non pas techniques, mais plus souvent humains ou organisationnels entraînant un taux d'insatisfaction fort élevé.

Nous avons retenu les caractéristiques d'une étude exploratoire pour réaliser ce mémoire car, très peu d'études jusqu'à maintenant ont été produites mettant en cause la gestion des ressources humaines dans les petites et moyennes entreprises (PME) . 


\section{CHAPITRE I}

\section{LA DÉFINITION DU SUJET}

Les économies du monde entier ont amorcé une époque où le perfectionnement des ressources humaines, y compris la formation, est un des facteurs qui, avec la rapidité des changements technologiques, devrait constituer la clé d'un avenir plus prometteur. Dans cet esprit, plusieurs éléments extérieurs viennent rappeler aux dirigeants d'entreprises que l'environnement devient de plus en plus turbulent. Ainsi, le mouvement de mondialisation des marchés pour l'un, ne laissera pas les administrateurs indifférents. Comme mentionne dans un article sur la planification des changements technologiques:

L'accord du libre-échange avec notre voisin du sud est maintenant acquis et son implantation déjà en cours. Dans 10 ans, peu d'entreprises pourront prétendre avoir échappé aux conditions nouvelles implantées par ce traité. Ces conditions nouvelles ne constituent d'ailleurs que quelques-uns des nouveaux défis que devront relever les entreprises dans le futur: pensons par exemple à l'élimination des quotas et à la déréglementation qui contribuent grandement à accroître la concurrence provinciale, nationale et internationale. Dans un contexte où le coût de la main-d'oeuvre augmente et celui d'un bon nombre de technologies décrốt, les experts s'accordent pour reconnaitre que les entreprises 
n'ont d'autres choix que de recourir pour améliorer leur position (Betcherman et Mc Mulien, 1986: Fréchet, 1987; Raymond 1987).1 à la technologie concurrentielle. Dusseault, 1985;

Ce thème qui est devenu une réalité de plus en plus grandissante a été repris par Julien, Carrière et Hébert qui mentionnait et je cite: "

La majorité des pays industrialisés éprouvent, depuis quelques années, de sérieux problèmes de productivité causés entre autres, par l'augmentation de leurs coûts en main-d'oeuvre, en matière première, et en énergie, par de nouvelles contraintes crées par la protection de l'environnement de leur appareil de production. ceci survient au moment même où ces pays ont à affronter simultanément une concurrence toujours plus vive sur les marchés domestiques et internationaux et un rétrécissement de ces mêmes marchés avec le ralentissement de la croissance démographique. 2

Ces deux situations contribuent à mettre en relief la nécessité de recourir aux nouvelles technologies et les mesures d'adaptation que les entreprises devront mettre en oeuvre. D'ailleurs, une étude du Conseil Économique du Canada entreprise sur le recentrage technologique mentionnait:

Gagnon, Yves-Chantal et Landry Maurice. La planification des changements technologiques et la survie des PME: réflexion sur un cas vécu. Revue PMO volume 5- No.2.

Julien, Pierre-André, Carrière, Jean-Bernard et Hébert, Louis. GREPME. La diffusion des nouvelles technologies dans trois secteurs industriels. UQTR. Conseil de la science et de la technologie, Avril 1988. 
Le Canada doit s'empresser d'adopter les nouvelles techniques afin de pouvoir maintenir et promouvoir la productivité, la qualité de vie, les capacités concurrentielles et l'emploi; il doit en même temps se doter d'un ensemble de politiques qui faciliteront cette transition tout en minimisant les couts. Ces deux éléments sont interdépendants: s'il n'accélère pas son recentrage technologique, le Canada perdra du terrain au plan de la concurrence et des emplois; s'il ne cherche pas en même temps à produire une main-d'oeuvre sûre, souple et hautement compétente, son progrès technologique se ralentira.

Ainsi, les entreprises doivent prendre le virage technologique compte-tenu du nouvel environnement qui devient de plus en plus turbulent et concurrentiel. Plusieurs études ont démontré, que bien que le Québec et le Canada aient fait de grands pas dans l'informatisation, dans la recherche et développement, un retard important persiste à cet égard avec d'autres pays avancés notamment le Japon, les États-Unis et l'Europe Occidentale.

Parmi ces études, un article paru dans la revue du marché du travail du MMSR mentionnait:

Les applications de la bureautique ont connu un rythme de diffusion accéléré au cours des années 80 . Par contre, les techniques de productique, dont les couts d'implantation sont généralement beaucoup plus élevés, n'ont pas bénéficié d'une aussi grande diffusion. Dans l'ensemble du secteur manufacturier, où se concentre la

Conseil Économique du Canada. Le recentrage technologique. Innovations, emplois, adaptations. 1987. p.2. 
grande majorité de ce type d'investissement, $43 \%$ des entreprises canadiennes ont fait état de changements technologiques liés à la productique entre 1980 et 1989.4

Un rapport récent financé par le Conference Board of Canada discourait sur ce thème en rapportant que:

Les organisations canadiennes ont clairement compris la relation critique entre les changements technologiques et la competitivité. Depuis les années 1980, le taux de diffusion des nouvelles technologies s'est accéléré dans les grandes entreprises. Selon 58 grandes entreprises syndiquées qui ont répondu à un questionnaire détaillé au printemps de 1990, toutes sauf deux avaient adoptée de nouvelles technologies au cours des cinq (5) dernières années et toutes ces entreprises sauf trois(3) prévoyaient d'autres changements au cours des cinq (5) prochaines.

Selon un sondage mené parmi 30 dirigeants d'entreprises syndiquées, tous ont mentionné que leurs intentions d'apporter des changements technologiques ou organisationnels avaient diminué significativement durant la présente récession et qu'ils ne prévoyaient pas d'améliorations à ce chapitre avant 1992; il faut mentionner que la croissance des entreprises ayant adopté de nouvelles technologies a cru dans les deux chiffres au Canada de 1985 à 1988 et de plus de cing 5 pour cent en 1989. Il est également apparu que les nouvelles technologies identifiées comme la clé d'une meilleure compétitivité par l'organisation Canadienne de Développement Economique (OCDE) ont donné le ton. Ainsi parmi les répondants qui ont fait l'acquisition de nouvelles technologies, 91 pour cent avaient eu recours à des appareils reliés à la productique, 68 pour cent avaient introduit des machines à contrôle numérique et

Béland, Pierre, Lalande Daniel et Roy, Normand. La formation professionnelle: les défis des années 1990 . Article dans la revue "Le marché du travail". Mars 1992 . 
64 \% avaient expérimenté des appareils de conception ou fabrication assistée par ordinateur. Seulement 11 pour cent ont rapporté avoir utilisé de l'équipement industriel avancé (biotechnologie) et 20 pour cent ont essayé l'intelligence artificielle. 5

Les grandes entreprises ont compris que, face à de sérieux problèmes de productivité causés entre autres par l'augmentation des coûts en main-d'oeuvre, en matières premières, par des nouvelles contraintes crées par la protection de l'environnement et une concurrence de plus en plus vive, qu'ils faillaient recourir aux nouvelles technologies. Il est vrai également que les grandes entreprises peuvent profiter de leurs moyens financiers pour se moderniser.

Dans une étude sur les facteurs de diffusion et de pénétration des nouvelles technologies dans les PME québécoises, les auteurs disaient à ce sujet que:

Il est vrai que de nombreux facteurs peuvent faciliter le passage des $P M E$ vers l'usine du futur. Ainsi les producteurs de ces équipements tendent de plus en plus à orienter leurs produits vers les besoins et les possibilités de celles-ci. De plus leur faible taille et leur flexibilité si souvent constatées les dégagent des problèmes de conversions coûteuses. Enfin, les PME

Wright. Ruth. Strategic Connections: Technology, Innovation and Labour Relations. A Conference Board of Canada Report from the Compensation research centre. March 1991. P.7. Traduction libre. 
se montrent plus souvent innovatrices que les grandes entreprises, augmentant d'autant plus leurs chances de s'adapter rapidement aux nouvelles technologies. cependant d'autres études montrent que plusieurs obstacles subsistent. Ils peuvent provenir du manque d'adéquation des nouveaux équipements pour les $P M E$ où de leur coût trop élevé, compte-tenu d'une capacité financière limitée où d'un haut niveau d'endettement. Ils peuvent également provenir du propriétaire-dirigeant qui manque souvent d'information sur les caractéristiques et les possibilités de nouvelles technologies.

Parallèlement, les PME ne possèdent pas toujours, au sein de son personnel, l'expertise nécessaire pour opérer une machinerie complexe. D'autres résistances couteuses peuvent provenir du personnel de l'entreprise qui, lui faute d'informations, comprend mal les motifs de modernisation et craint de voir disparaitre des emplois. ${ }^{6}$

Donc l'adoption de nouvelles technologies par les PME ne se limite pas à l'acquisition de l'équipement. Plusieurs études ont démontré que ce recours a modifié la structure de l'emploi (beaucoup d'emplois disparaissent, d'autres naissent et d'autres changent de nature), les qualifications professionnelles et l'organisation du travail. D'ailleurs sur ce sujet l'étude du conference Board cité précédemment mentionnait:

Julien P.A., Carrière, J.B. et Hébert L. Les facteurs de diffusion et de pénétration des nouvelles technologies dans les PME québécoises. GREPME. UQTR. Sept.1987. P. 3 . 
Ce n'est pas tout simplement suffisant d'acheter à l'étranger de l'équipement. L'acquisition, selon l'OCDE, ne se limite pas seulement à l'investissement monetaire mais doit prendre en note d'autres considérations dans l'utilisation efficiente de la nouvelle machine. Ainsi, la ré-organisation du travail, les pratiques de gestion, la formation, l'acquisition d'habilités et les relations de travail à améliorer sont le "software" qui conduit à la technologie."

Bref, la concurrence oblige les entreprises à s'adapter et à recourir à de nouvelles technologies en particulier celles axée sur la micro-électronique. Ce recours ne se fait pas sans heurts et de nombreuses adaptations se sont produites dans ces entreprises pour faire face aux innovations adoptées de sorte que certains employés ont été mutés à de nouveaux postes, tandis que d'autres ont dú se recycler pour acquérir de nouvelles compétences.

Je me propose dans ce mémoire d'étudier la dimension humaine du changement technologique. Plus précisément, je veux m'arrêter aux activités reliées à la formation du personnel suite à l'adoption de nouvelles technologies par les PME. 
CHAPITRE II

PROBLEMATIQUE

La nécessité de recourir aux nouvelles technologies étant définie, plusieurs entreprises se sont rapidement tournées vers l'adoption de celles-ci sans par contre obtenir les résultats escomptés. Ainsi, dans l'article publié par le Conference Board, il est mentionné que:

Les expériences tenues plus tôt avec la technologie reliée à la micro-électronique ont enseigné aux employés et à la direction de rigoureuses leçons. Une mauvaise planification, un choix inapproprié dans l'équipement et, par dessus tout, l'insensibilite de la direction envers les effets défavorables sur les employés se sont traduits par un mouvement de recul de la part des firmes canadiennes. Une étude américaine prétend que 50 à 75 pour-cent des entreprises qui ont fait l'acquisition de nouvelles technologies ont connu des ratées . Carol Beatty de l'université Queen a découvert que la moitié des entreprises qui ont participé à une étude sur la fabrication ou la conception sur ordinateur ont eu des expériences non satisfaisantes. Les problèmes rencontrés étaient fréquemment humains et organisationnels plutôt que techniques mentionna Beatty et la moitié des échecs pouvaient être directement reliés à une mauvaise gestion des ressources humaines. 
Ce lien entre l'adoption des nouvelles technologies et l'insatisfaction dans les résultats obtenus a été repris par plusieurs auteurs. De plus, plusieurs études ont fait part également de l'aspect humain qui prend une place des plus importantes dans l'adoption des nouvelles technologies.

Ainsi, entre autres, Ann Majchrzak qui a passé plus de sept années dans une recherche et des consultations sur le sujet en cause mentionnait dans son volume:

Malgré l'urgence de prendre le virage technologique dans le secteur manufacturier, les firmes américaines connaissent pour le moment un taux d'échec estimé entre 50 et 75 pour cent lors de l'implantation de nouvelles technologies (Ettlie, 1986 a; Hawang et Salvendy, 1985; Jaikumar, 1986) 9

Une des raisons expliquant un tel résultat serait reliée aux pratiques de management dont l'aspect des ressources humaines a été identifié comme le facteur crucial dans le succès et l'implantation effective de technologies avancées.

Dans son volume, Mme Ann Majchrzak cite plusieurs recherches récentes qui confirment la nécessité de la The Jossey-Bass management series. 1988. P. XI. Traduction libre. 
planification des ressources humaines dans l'adoption de nouvelles technologies. Parmi celles-ci, on mentionne que:

Le Congressionnal office of the Technology Assesment a mené en 1984 une étude impliquant des enquêtes de cas sur l'implantation d'ateliers flexibles. L'étude a conclu que la principale pierre d'achoppement pour I'implantation future d'automates programmables n'est pas technique, mais plutôt des barrières de coûts, un manque de compétences appropriées et une méconnaissance des effets sociaux de l'application des nouvelles technologies loffice of Technology Assessment, 1989, p.94). Dans une étude de l'Académie des sciences dans laquelle vingt-quatre (24) ateliers ayant implanté des automates programmables ont été visités, les conclusions étaient les mêmes: les changements non-techniques alliés aux nouvelles technologies causaient bien souvent de plus grands problèmes que la technologie elle-même (Manufacturing Studies Board, 1986). 10

De même, le Conseil Économique du Canada dans son étude sur le recentrage technologique discourait sur deux éléments, soit l'adoption rapide des innovations technologiques et des mesures d'adaptation visant à produire une main-d'oeuvre qualifiée, dans une stratégie d'adhésion aux technologies de pointe.

Pour répondre à la stratégie citée précédemment, le Conseil Économique émettait cinq (5) objectifs généraux qui devenaient en quelque sorte les conditions de réussite de l'implantation des nouvelles technologies qui sont: 
1. Pour accrôtre la productivité, la capacité concurrentielle, les revenus et le nombre d'emplois, le Canada doit s'ouvrir d'emblée aux mutations technologiques...

2. Si vitales qu'elles soient, les innovations technologiques ne suffiront pas à réaliser les améliorations que nous souhaitons. La présence d'une main-d'oeuvre qualifiée, souple et enthousiaste aura un rôle tout aussi important à jouer.

3. Les transformations de la technologie se produisent à un rythme irrégulier et engendrent des ruptures. II faut mettre en place une politique d'adaptation qui puisse en réduire les coûts au minimum et assurer que ses bienfaits seront partagés équitablement.

4. Pour assurer le succès du recentrage technologique, les Canadiens devront innover sur deux plans. La réussite dépendra tout autant des innovations apportées aux structures du travail et de la mise en valeur des ressources humaines que des connaissances techniques.

5. Pour que les Canadiens puissent jouir des bienfaits du progrès technologique dans un contexte de concurrence internationale acharnée, ils doivent instaurer un climat plus positif dans le domaine des relations du travail. 11

Donc, une nouvelle fois, plusieurs facteurs de réussite pour l'adoption des nouvelles technologies sont avancés dont le style de gestion, la mise en valeur des ressources humaines, le recyclage et la formation continue, un climat de travail positif, etc... Appliqués aux grandes entreprises, ces conditions ou facteurs de réussite peuvent y trouver un écho compte tenu des moyens financiers et techniques s'y attachant. De même dans une étude entreprise par le centre de 
recherche et de statistique sur le marché du travail au printemps 1991 portant sur les changements de l'organisation du travail dans le contexte de la mondialisation économique, les résultats sur les mesures pouvant favoriser l'introduction de ceux-ci allaient dans le même sens que les facteurs énumérés ci-haut. Ainsi, on mentionnait que:

Une information plus complète sur le processus d'introduction de changements dans l'organisation du travail, des cours de formation professionnelle mieux adaptés aux besoins des entreprises et une plus grande participation du syndicat constituent trois éléments qui, selon les répondants, devraient faciliter l'introduction des changements dans l'organisation du travail. $\underline{12}$

Cette enquête avait été menée auprès de 404 établissements au Québec dont 202 questionnaires ont été retournés mais provenant d'entreprises de 75 salariés et plus.

A partir de ces constatations, je m'attarderai à l'aspect de la formation dans les PME. Ainsi, la planification par les entreprises de leur besoin en maind'oeuvre et de formation constitue un problème majeur et mois. Les changements de l'organisation du travail dans le contexte de la mondialisation économique. Aout 92.P.10. 
plusieurs études sur ce sujet en font mention. Cette planification est souvent insuffisante, les entreprises faisant preuve de courte vue et se limitant au coup par coup, et ce même après l'arrivée de nouveaux équipements.

Dans une recherche menée pour le compte du Ministère de la Main-d'oeuvre de la Sécurité du Revenu et de la Formation Professionnelle, les auteurs abordent plusieurs aspects reliés à la gestion des ressources humaines dans les PME dont la formation. Sur cette dernière activité, elles confirment entre autres que les dirigeants pensent en majorité que la responsabilité du perfectionnement du personnel de leur entreprise leur revient d'abord. Fort surprenant, $85 \%$ affirment que cette responsabilité ne revient pas du tout au syndicat. Par ailleurs, chez les entreprises syndiquées, 64 $\%$ ne reconnaissent aucune responsabilité au syndicat alors que $36 \%$ le font à divers degrés.

Certains faits qui ont été observés par cette étude sont révélateurs sur la formation dans les PME. Ainsi, parmi les éléments figurant dans la conclusion se rapportant au sujet qui nous intéresse, il est mentionné que:

Les entreprises disent pourtant poursuivre de nombreux objectifs de changements, et ces objectifs génèrent 
d'importants besoins de formation pour toutes les catégories d'employés, mais ces besoins ne se traduisent pas par des efforts suffisants de formation.

En effet, $26.0 \%$ des entreprises n'ont fait aucune formation, 28, $0 \%$ n'ont offert que de la formation en cours de travail et $46,0 \%$ de la formation en cours de travail et de la formation organisée, le genre de formation le plus généralement répandu étant l'entraînement à la tâche (87.8\%). En général, les dirigeants préfèrent embaucher du personnel déjà formé, même s'ils rencontrent de sérieux problèmes de recrutement de personnel qualifié.

L'importance de la formation progresse avec la taille des entreprises. Seulement $27 \%$ des employés dans l'ensemble des entreprises ont eu réellement accès à la formation en cours de travail et $14 \%$ à la formation organisée.

Les entreprises recourent peu à des ressources externes pour résoudre leurs problèmes de formation que ces ressources soit des consultants, des établissements publics ou privés ou des programmes gouvernementaux. Les entreprises qui font de la formation organisée pendant les heures de travail rencontrent des obstacles importants, tels le manque de temps et de personnel requis pour planifier et organiser la formation, de même que la difficulté de concilier la planification de la planification et celle de la production, les PME étant des entreprises sensibles aux fluctuations de la demande. $\underline{13}$

Bref, la gestion de la formation ne semble pas occuper une place prépondérante dans les activités des entreprises

Benoit, Carmelle et Rousseau. Marie-Diane. La gestion des ressources humaines dans les PME au Québec. M.M.S.R.F.P. Mai 1992.P.180-183. 
même si les dirigeants affirment en prendre conscience. La fonction formation est encore perçue comme une dépense et non un investissement à long terme. De plus, lorsqu'on dispense de la formation, la formation sur le tas semble la plus répandue. Pourtant ce type de formation ne semble pas répondre à toutes les attentes. Ainsi, dans un article sur la formation professionnelle dans les années 1990, les auteurs mentionnent:

Même s'il a toujours sa place l'apprentissage sur le tas s'avère nettement insuffisant lorsque les entreprises entreprennent un processus de modernisation. Dans plusieurs cas, les employés qui ont acquis un savoirfaire empirique doivent acquérir des connaissances plus théoriques, nécessitées par les nouveaux procédés de travail. Les gestionnaires qui ont à gérer des changements dans l'organisation du travail sont souvent des contremaitres choisis parmi les travailleurs de production. Ceux-ci doivent apprendre à gérer les transformations importantes dans les procédés de production, s'adapter à un contexte de travail qui fait place à une plus grande participation des travailleurs et améliorer leurs propres compétences en gestion des ressources humaines. $\frac{14}{4}$

Par ailleurs, au sujet de l'effort actuel de formation par les entreprises québécoises, la source d'information la plus complète sur le sujet est l'Enquête sur la formation et le développement des ressources humaines, que statistique Canada a réalisé auprès des établissements pour le compte 
d'Emploi et Immigration canada. ${ }^{15}$ cette enquête a révélé qu'en 1986-87, les entreprises ont dépensé 1,4 milliard de dollars, soit approximativement $0,6 \%$ de la masse salariale, ce qui équivaut à 160\$ par salarié et représente moins de la moitié des frais de formation engagés par les entreprises américaines. ${ }^{16}$

Parmi les différentes constatations de l'enquête, seulement $31 \%$ des entreprises offrent effectivement de la formation structurée à leurs employés. La formation est relativement peu courante dans les petites entreprises: $27 \%$ des entreprises comptant moins de 10 employés en offrent, comparativement à $76 \%$ des entreprises de 100 employés ou plus et à 92\% de celles comptant 1000 employés ou plus.

Selon les études de la Fédération canadienne de l'entreprise indépendante (FCEI), la situation irait en s'améliorant. Selon cet organisme $70 \%$ des 83,000 firmes canadiennes membres font de la formation et ce en tenant compte de celle faite sur les lieux de travail. Selon un

15 Voir Statistique Canada. Enquête sur la formation et le dèveloppement des ressources humaines. 1989

Voir Emploi et Immigration Canada. Le nouveau mode d'emploi: profil de la croissance du marché du travail.1989. 
article paru dans la revue Les Affaires du 17 Octobre 1992, M.Decary, vice-président de la Fédération mentionnait que: "nous n'avons pas les ressources pour perdre des employés alors qu'ils sont en formation à l'extérieur. Quand vous ne comptez que trois ou quatre employés, cela devient très difficile."

Par contre, le peu de formation dispensée aux employés peut s'avérer un frein important à l'utilisation maximale de la nouvelle machinerie, en particulier celle reliée aux nouvelles technologies. Ainsi dans un article paru dans la revue "Les Affaires", M.Benôt Tremblay, directeur de gestion de la qualité pour Lar Machinerie, une entreprises de moins de 200 employés oeuvrant dans l'usinage au Lac st-Jean, a décidé de recourir à une firme de formation de l'extérieur pour dispenser le programme de formation. Avec la récente acquisition d'une machine-outil à commande numérique, l'entreprise s'est retrouvée avec un équipement qui lui procurait peu d'avantages, à défaut d'en comprendre toutes les possibilités. Le fournisseur s'était contenté de démarrer la machine pour rendre les employés tout juste fonctionnel. ${ }^{17}$ 
A partir de ce dernier exemple, je me propose dans ce mémoire de mettre l'accent comme mentionné auparavant sur la dimension humaine $d u$ changement technologique. Plus précisément, je veux porter la recherche sur les conditions de succès reliées aux activités de formation dans les PME faisant face à l'adoption d'équipements reliés aux nouvelles technologies. Plusieurs études démontrent certaines conditions assurant le succès dont entre autres, la participation des employés, le dialogue avec le syndicat, la formation continue avec le personnel, etc. Je vise comme objectif d'aller vérifier auprès d'entreprises ayant eu recours à ces nouvelles technologies, les étapes à franchir et les écueils à éviter pour obtenir le succès et la rentabilité.

Cette étude portera sur un nombre limité d'entreprises oeuvrant dans le secteur des ateliers d'usinage au Saguenay/Lac st-Jean. Les résultats, compte tenu du nombre, ne pourront permettre de généraliser mais pourront s'avérer un excellent outil pour la compréhension du rôle important de la formation des employés sous l'angle d'un investissement. 
CHAPITRE III

LE CADRE THÉORIQUE

Dans les pages qui suivent, je vous tracerai le cadre théorique dans lequel s'inscrira cette recherche ainsi que l'explication du champ de connaissances dans lequel s'insère le problème ainsi que ces limites.

cette recherche s'inscrit donc dans le cadre des théories reliées aux sciences administratives et plus particulièrement à celles s'orientant vers la gestion des ressources humaines. Cette option a fait l'objet au cours des dernières années de nombreuses publications fondées sur des études empiriques décrivant les pratiques et les systèmes de GRH adoptés par les entreprises. Dans l'ensemble les études empiriques et théoriques sur la GRH dans la grande entreprise ont permis de mettre en lumière la variété des pratiques et de relier les réalités à un ensemble de facteurs déterminants tels: l'environnement de l'entreprise, la stratégie d'entreprise, la culture de l'entreprise et la vision du dirigeant. 
$\mathrm{Au}$ niveau des $\mathrm{PME}$, les études sur le sujet révèlent que la vision du dirigeant, ses valeurs et ses objectifs jouent un rôle prédominant dans la conception et les réalisations des activités de GRH selon les recherches menées par Mahé de Boislandelle. ${ }^{18}$ De même cet auteur mentionnait que la place occupée par les ressources humaines comme l'objet d'étude demeure très limitée et marquée par une absence d'analyse et d'outil approprié à la petite et moyenne entreprise.

Tenant compte de ce contexte, j'ai adopté le modèle conceptuel présenté par Madame Ann Majchrzak intitulé "The Human Infrastucture Impact statement (HIIS)" qui a été élaboré suite à de nombreuses recherches et études menées auprès de différentes entreprises sur le sujet. Selon ce modèle des problèmes de gestion et de direction surviendront si une stratégie d'adoption de nouveaux équipements n'est pas planifiée et assumée. L'approche adoptée illustre non seulement le besoin de planification mais également les effets interactifs du changement sur l'équipement, la structure, le travail et les gens. ressources humaines dans les PME. Edition Economica. 1988 . 
Pour en arriver à èlaborer ce modèle, madame Majchrzak débutait sa recherche en discourant sur le lien entre la planification des ressources humaines et le succès obtenu dans l'adoption de nouvelles technologies. Ainsi, elle mentionnait que le peu d'attention consacrée aux ressources humaines a conduit à une série d'affirmations se rapportant au facteur humain. Parmi celles-ci, nous observions les suivantes:

- l'utilisation optimale de la machinerie peut être atteinte par le recours à des facteurs techniques.

- les travailleurs sont plus flexibles que l'équipement ou la machinerie.

- l'impact sur les ressources humaines de l'utilisation de nouvelles technologies peut facilement être circonscrit par le recours à des politiques de gestion du personnel consistant à réécrire les descriptions des postes de travail ou en dispensant de la formation; les autres effets de l'implantation de technologies étaient inconnus ou trop difficiles à prévoir. 
Dans bien des cas, selon Ann Majchrzak ces affirmations pouvaient s'avérer exactes lors de l'achat d'équipement conventionnel. Dans le passé, l'implantation de technologie était souvent une affaire simple. La fiabilité de la machinerie était prévisible, les fonctions reliées à l'utilisation et à la gestion du nouvel équipement étaient bien définies et les principales politiques se rapportant à la gestion des ressources humaines, comme l'échange d'information et la coordination des divers départements et services, étaient rarement modifiées avec la nouvelle technologie. Ainsi, les problèmes rencontrés requéraient bien souvent des solutions techniques. Le seul type de solution se rapportant au facteur humain se référait à l'élimination de coût direct en main-d'oeuvre. Une fois la décision prise d'acheter l'équipement, aucune marge de manoeuvre n'était permise, les changements dans le personnel étaient plus facilement prévisibles que ceux se rapportant à la machinerie. Ainsi, la planification des changements sur le personnel semblait beaucoup moins importante que celle reliée à la technologie. En outre, vu la plus grande flexibilité dans la gestion des ressources humaines obtenue en réécrivant les fiches de poste et en formant le personnel l'ajustement de ces facteurs humains devenait la priorité. 
Compte tenu que l'implantation de l'équipement conventionnel a montré la voie à suivre pour celle reliée aux nouvelles technologies, plusieurs entreprises ont continué d'ignorer l'impact sur les ressources humaines jusqu'à la mise en marche de la nouvelle machinerie. Madame Majchrzak dans son volume fait référence à plusieurs études effectuées dans le cadre de l'implantation de nouvelles technologies. Parmi celles-ci, on mentionnait que plusieurs dirigeants on rapporté un manque dans les considérations initiales se rapportant aux conséquences de l'automatisation sur l'aspect humain (Butera, 1894). Les gestionnaires ont indiqué que les ressources humaines étaient considérées seulement en dernier recours pour résoudre des problèmes s'il y a lieu. D'autre études récentes menées dans plusieurs industries manufacturières ont aussi mentionné le manque de considération apportée à l'infrastructure humaine lors de l'implantation de nouvelles technologies. (Bishop, 1984; Blumberg and Gerwin, 1985; et all.) En outre, lorsqu'on prenait en considération les conséquences humaines de l'introduction de nouvelles technologies, l'étude s'arrêtait bien souvent à la réduction des coûts de main-d'oeuvre (Ayres et Miller, 1983). L'auteur dans son volume démontre à l'aide de plusieurs exemples qu'à défaut de prévoir l'impact sur les infrastructures humaines d'un changement dans la 
machinerie relié à une nouvelle technologie, les entreprises courent au devant des problèmes. Parmi ceux-ci, l'assertion voulant que l'utilisation optimale de la machinerie peut être atteinte par le recours à des facteurs techniques, n'a jamais été supportée par les recherches sur le sujet. Bien plus, au contraire, la principale pierre d'achoppement suite à l'adoption de nouvelles technologies n'était pas technique, mais reliée à des barrières de coût, d'organisation de l'usine, de compétences et habilités appropriées ainsi que des effets sociaux.

Plusieurs exemples tirés d'entreprises ayant adopté de nouvelles technologies sont énumérés et mettent en lumière les difficultés rencontrées au chapitre des ressources humaines dans l'étude de madame Majchrzak. Par ailleurs, tous en viennent à la conclusion qu'ignorer les ressources humaines jusqu'au moment où la machinerie est installée amènera des maux de tête pour les dirigeants dans la gestion des ressources humaines. Ces problèmes peuvent être assez sérieux au point où le capital investi dans de nouvelles technologies pourrait être tout simplement annulé. Bref, les compagnies ne peuvent pas effectivement adopter de nouvelles technologies sans la planification de l'infrastructure humaine. 
Avant d'aller plus loin dans l'étude et la présentation du modèle, il convient de bien définir les concepts. Lorsque tout au long de ce mémoire nous faisons référence à la nouvelle technologie, il faut se rapporter à la conception assistée par ordinateur, au centre d'usinage, aux ateliers flexibles, etc.... Pour la définition de nouveaux équipements de production, les auteurs Julien et Thibodeau fournissent une liste et une description divisée selon le niveau de complexité pour les industries du secteur primaire et secondaire; ce dernier est le secteur où évolue les ateliers d'usinage. Les lecteurs en retrouveront le contenu en annexe I. Pour les activités de formation, plusieurs définitions existent. J'ai retenu la suivante:

La formation est un ensemble d'activité d'apprentissage planifié visant l'acquisition de connaissances, d'habilités et d'attitudes propres à faciliter I'adaptation des individus et des groupes à leur environnement socio-professionnel et en même temps, la réalisation des objectifs d'efficacité de I'organisation. 19

Ayant défini le champ de connaissances et des limites, rien ne nous empêche de s'attarder maintenant au modèle 
conceptuel développé par Ann Majchrzak et à l'outil appelé HIIS dont le but est :

Le HIIS est une outil servant à analyser l'infrastructure humaine des organisations avant l'achat d'équipement pour déterminer quels impacts pourraient vraisemblablement survenir et par conséquent quels choix devraient-on effectuer dans l'équipement et l'organisation humaine afin d'atteindre le plus efficacement possible le but poursuivi. $\underline{20}$

Selon l'auteur, l'adoption de nouveaux équipements est faite en fonction de deux buts soit pour assurer la survie de l'entreprise soit pour procéder à des changements dans le processus de production afin de maintenir la croissance et la survie de l'entreprise. Les bénéfices de la survie organisationnelle de l'entreprise peuvent inclure la croissance des ventes dans un marché existant, l'entrée dans de nouveaux marchés ou juste rester au même point. Les bénéfices découlant de l'autre option peuvent inclure la réduction des rebuts, l'utilisation maximale de l'équipement, une réduction du taux de rotation et autres...D'autres gains peuvent provenir de la satisfaction du personnel, d'une diminution de l'absentéisme et du taux de roulement et une baisse des grèves et des accidents de travail. 
Par ailleurs, tous les avantages mentionnés n'arrivent pas systématiquement suite à l'adoption de nouveaux équipements. Ces résultats positifs surviennent suite à un processus de changement dans l'infrastructure humaine dont par exemple une meilleure attention de la direction aux résultats de la performance des divers départements, une meilleure collaboration entre les opérateurs d'équipement et les ingénieurs afin de réduire les bris, etc.

Le modèle développé par L'auteur comprenant les six principales composantes est présenté en annexe II. Cellesci sont présentées par étape signifiant que les premières décisions auront de l'influence sur celles prises par la suite. Ainsi les étapes incluent le choix de l'équipement, les effets qu'auront ces décisions sur les emplois (premier effet), les répercussions de ces dernières sur la formation du personnel, la sélection et les autres politiques de personnel (second effet) et enfin les résultats finaux de l'adoption de nouvelles technologies sur le processus de production, les bénéfices sur l'infrastructure humaine et la survie ou les buts recherchés par l'entreprise. Les deux autres composantes sont le processus de changement pour implanter les décisions et les contraintes. 
Au sujet du choix de l'équipement, l'auteur mentionne que celui-ci doit être en harmonie avec l'infrastructure humaine. Ann Majchrzak a développé une typologie au sujet des paramètres se rapportant à l'équipement que le gestionnaire doit prendre en considération. Bien qu'intéressant, nous allons nous arrêter qu'à les identifier sans plus car nous déborderons des buts poursuivis par le sujet de ce mémoire. Ceux-ci sont: l'intégration, la rigidité, la fiabilité, la rétro-action, ainsi que la sécurité.

Concernant les effets de premier ordre, le choix de l'équipement se répercutera directement sur les emplois des opérateurs de la machinerie, ceux du personnel de support technique et administratif ainsi que ceux reliés à la direction et supervision.

Pour les opérateurs de machine, l'adoption de nouvelles technologies pourrait se traduire par des besoins nouveaux en information et en coordination. De même, le personnel pourrait être appelé à exercer certaines tâches routinières comme l'ajustement de la machinerie avant chaque opération ou de presser sur les boutons de départ et de fin et ce, de façon continuelle. De même, son nouvel emploi pourrait 
l'amener à avoir plus de relations avec le personnel technique ou d'entretien et les ingénieurs. Bref, ses tâches pourraient être modifiées.

De la même façon, le personnel technique et de soutien pourrait voir leurs définitions de tâches se modifier comme par exemple de confier le contrôle de qualité de la production à l'opérateur de l'équipement plutôt qu'à eux. Les superviseurs et la direction peuvent être également touchés. Comme par exemple, la haute direction, suite à la nouvelle technologie, pourrait consacrer plus de temps à l'identification de nouveaux marchés pour les produits qu'auparavant.

En résumé la description de ces premiers effets pourrait se traduire par des changements dans les fiches de postes d'une partie du personnel de l'usine. Ainsi, l'auteur en vient à introduire la série de deuxième effet qui n'est pas reliée à l'équipement ou la machinerie mais aux décisions qui seront prises suite aux effets vus précédemment.

Les effets qui impliquent des changements dans l'infrastructure humaine peuvent se traduire par des habilités à acquérir, des activités de sélection et de 
formation du personnel et les politiques de gestion du personnel.

Bien qu'une fois que l'équipement aura été choisi et les effets sur l'infrastructure humaine auront été planifiées, il faut également apporter une attention au processus de changement.

Voulant introduire une nouvelle technologie, les dirigeants devront garder à l'oeil quatre éléments. Dans un premier temps, il faudra apporter une attention à la résistance aux changements de la part du personnel, surtout si l'équipement entraîne des modifications chez les travailleurs. La direction devra également prévoir des actions pour contrer la résistance dont ses actions visant l'éducation, la participation et la mobilisation des employés. Troisièmement, la création de groupes de travail ayant la responsabilité de la sélection de la nouvelle technologie et son implantation pourrait s'avérer un gage de succès. Finalement, le processus de changement doit se faire par étapes où les considérations du personnel sont prises en compte plutôt que d'obliger les employés à faire face à l'arrivée d'une machinerie avec des changements dans 
l'organisation du travail et ce, une fois toutes les décisions prises.

La dernière composante du modèle est une série de contraintes qui influencent les décisions reliées à l'infrastructure humaine. Aucune organisation n'opère dans le vide; ce serait une erreur que de vouloir analyser les effets sur l'infrastructure humaine sans tenir compte de ces contraintes. Quatre contraintes ont été identifiées comme ayant des effets particulièrement importants sur les divers choix soit: la force du travail, la planification du processus, la prévision du marché et l'importance accordée par la gestion aux ressources humaines. Une vue d'ensemble du modèle développé par l'auteur vous est présentée en annexe II.

Pour chaque contrainte, nous devons également s'interroger sur les effets que l'adoption de nouveaux équipements pourraient avoir. Ainsi, pour la force de travail, les impacts peuvent être examinés en analysant les quatre caractéristiques suivantes soit:

- L'intérêt des travailleurs à relever des défis. 
- Le degré de confiance des travailleurs envers la direction. Si les employés se font mentionner qu'il n'y aura pas de mises à pied, cette affirmation ne sera pas prise en considération si le degré de confiance est à son plus bas. Dans ce cas, la motivation des employés à rendre opérationnel l'équipement le plus rapidement possible sera pratiquement inexistante. Dans le cas contraire, où la direction détient la confiance du personnel, une économie de temps significative sera réalisée dans l'information et la planification du processus.

- La présence d'un syndicat. Dans les entreprises syndiquées, le processus de changement doit-être discuté avec le représentant des travailleurs, tant local que national, et les nouvelles politiques du personnel et les fiches de poste doivent être renégociées s'il y a lieu.

- Le niveau de compétence des travailleurs. Si les travailleurs sont déjà très qualifiés, l'adoption de nouveaux équipements pourraient poser des problèmes advenant que le personnel soit relégué à des tâches de routine demandant peu d'initiative. Dans le cas contraire, les travailleurs routiniers exigeront plus de formation pour être capable d'opérer comme il se doit la machinerie avec des 
responsabilités et une prise de décision grandissante. De plus, il faudra tenir compte de l'expérience des superviseurs dans la gestion des opérateurs d'équipement. Des programmes de formation devront être planifiés également pour les gestionnaires. Enfin, il faudra tenir compte que le peu de sensibilisation du personnel et de la gestion avec la nouvelle technologie pourra commander un rythme plus lent d'introduction afin de pouvoir construire la base d'expérience nécessaire.

Pour la planification du processus, trois contraintes $s$ o $t$ identifiées soit:

- le degré d'autonomie sur le processus des différentes directions. Dans le cas des grandes entreprises, il pourrait survenir des changements dans la distribution des revenus d'un groupe (rémunération basée sur le rendement du groupe plutôt qu'une rémunération individuelle). Par contre, il faudra s'assurer qu'une telle décision puisse être considérée par le directeur du département et vue comme une option de la part des dirigeants.

- les accords avec les fournisseurs. A mesure que les fournisseurs pourront livrer les pièces à temps et avec un 
taux de rejet nul, plus fortes seront les probabilités que l'usine puisse atteindre de hauts niveaux de performance avec son équipement.

- la mobilité des travailleurs. Les occupations et les industries qui offrent plusieurs choix d'emploi exigent un processus de planification des ressources humaines. Comme une usine peut également s'attendre à un fort taux de roulement (et ainsi reconsidérer l'importance de l'investissent en formation dans les employés à court terme, ou apporter beaucoup de soin à son personnel pour les persuader à demeurer).

Au niveau des contraintes, il faut également tenir compte des impacts sur la prévision des ventes ou de l'évolution des marchés dont:

- la capacité des organisations de pénétrer rapidement de nouveaux marchés. La production a été automatisée afin d'aller chercher de nouveaux marchés et les dirigeants étaient tellement sûrs d'eux qu'ils ont fait des promesses de sécurité d'emploi au personnel. Une mauvaise prévision a fait que les résultats escomptés ne surviennent pas, il 
s'ensuivra alors une méfiance entre le syndicat et l'employeur qui dégénèrera sur le climat de travail.

- la non-atteinte des prévisions. Ce facteur peut créer souvent un sentiment d'urgence dans l'adoption des nouveaux équipements. Quand la non-atteinte des prévisions est clairement expliquée aux employés pendant la planification du changement, la situation d'urgence peut s'avérer d'un grand secours pour l'acceptation du processus.

- la prévision des croissances des ventes. L'implantation de nouveaux équipements peut impliquer graduellement la formation $d u$ personnel et la reconstruction de fiches d'analyse de poste afin de donner plus de responsabilités aux travailleurs. Comme une stratégie de croissance basée sur le long terme peut s'avérer très changeante dans un environnement turbulent, dans ce cas le modèle doit pouvoir compter sur une flexibilité et des changements rapides en cas de besoin.

Enfin, la philosophie de gestion est notre dernière contrainte à prendre en considération soit: 
- le modèle de motivation implanté dans l'entreprise. Le dirigeant se situe-t-il plus du coté $x$ de la théorie de Mc Gregor ou bien plus du coté Y?

- la prise de décision. La prise de décision appartientelle au propriétaire ou les superviseurs de première ligne peuvent-ils donner leurs idées?

- la priorité accordée aux ressources humaines. La formation peut s'adresser à l'ensemble du personnel ou à seulement quelques employés dont la formation est absolument nécessaire et constitue un seuil minimal.

Donc dans la pré-planification des ressources humaines à 1'adoption de nouveaux équipements, chacune des contraintes doit être considérée. Cependant chaque contrainte n'est pas nécessairement d'une importance égale ni immuable. Dans la planification $d u$ changement, ces contraintes doivent être le premier aspect de l'organisation à tenir compte et à modifier et ce, préalablement aux choix de l'équipement et à la prise de décision sur l'ensemble du personnel. Pour le chercheur, aucune étude des effets sur le changement technologique ne peut être conduite sans une compréhension du rôle que ces 
contraintes ont pu jouer sur les options de l'infrastructure humaine et les choix faits, selon l'auteur.

Finalement, dans le modèle développé par madame Majchrzak, l'infrastructure humaine est composée de onze éléments soit:

1- les activités reliées aux opérateurs de la machinerie;

2- les fonctions de support technique;

3- les fonctions de management;

4- les fonctions de supervisions;

5- les habilités requises;

6- la sélection;

7- les activités de formation;

8- les politiques de personnel;

9- la structure organisationnelle;

10- les résultats désirés;

11- le processus de changement mis en place.

Aux fins de ce mémoire, nous avons retenu l'élément se rapportant aux activités de formation. 
CHAPITRE IV

CADRE MÉTHODOLOGIQUE

La recherche scientifique se définissant comme une investigation ou une démarche systématique, contrôlée, empirique et critique d'une proposition hypothétique, son objectif vise donc à obtenir des réponses à diverses problèmes. Dans le cadre de ce mémoire, l'hypothèse est que l'adoption de nouvelles technologies doit se faire en n'ignorant pas les effets sur les ressources humaines à défaut de ne pas obtenir les résultats escomptés. Compte tenu du modèle conceptuel développé par madame Majchrzak, j'ai retenu un des éléments qui composent l'infrastructure humaine, soit la formation. Pour exercer les activités de formation, les gestionnaires doivent prendre en considération le facteur humain dans le cadre de l'adoption de nouvelles machineries. Selon le modèle présenté par Ann Majchrzak, quatre composantes doivent être prises en compte afin de s'assurer que l'entreprise atteigne le but fixé par son adoption. La recherche et les consultations menées par l'auteur à ce sujet ne s'adressaient pas nécessairement à des petites et moyennes entreprises. L'enjeu sera donc de 
vérifier si les mêmes conclusions prévalent dans les PME oeuvrant dans le domaine de l'usinage au Saguenay Lac stJean.

Compte-tenu de la nouveauté de sujet à l'étude, soit la prise en considération du facteur humain suite à l'évolution socio-économique des années 1960-80, et le peu d'études ayant été produites s'appliquant à la gestion des ressources humaines dans les PME, cette recherche épousera les caractéristiques d'une de style exploratoire et sur le terrain telles que définies par le modèle intégré de recherche (MIR) présenté par différents auteurs. ${ }^{21}$

ce type de recherche a aussi ses limitations. Ainsi, la recherche exploratoire permet d'aborder de façon globale et réaliste des champs de recherche encore inexplorés mais n'autorise pas de conclusions définitives. D'ailleurs, compte-tenu également que la réalisation des études d'impacts des changements technologiques pose de sérieux problèmes d'ordre méthodologique en raison de la complexité du phénomène que l'on cherche à mesurer, quelques études André-Jean. Comprendre l'organisation; approches de recherche.Les Editions Agence d'Arc Inc. Montréal.1983, $198 \mathrm{p}$. 
recensées sur ce sujet ont privilégié également les études de cas. $^{22}$

Le choix des entreprises devant faire l'objet d'études de cas a été fait selon les critères suivants:

- nous avons tout d'abord constitué la population totale des entreprises d'usinage au Saguenay Lac st-Jean. Ainsi, selon le relevé des employeurs, 78 entreprises embauchant 1221 employés(es) $y$ sont dénombrés. ${ }^{23}$ Toutes les entreprises font partie de la catégorie de PME, aucune n'embauchant plus de 200 employés(es).

- comme les études de cas visaient les entreprises ayant adoptées de nouvelles technologies, une demande d'identification a été adressée à tous les centre d'emplois

Voir: Nyahoho, Emmanuel. Impact des changements technologiques dans les secteurs d'activité économique du Québec et évolution structurelle de l'emploi. Revue le marché du travail vol.7, numéro 5. Mai 1986

Larouche, Gabriel. L'impact des machines-outils à commandes numérique sur l'emploi et les ressources humaines de l'entreprise. Revue le marché du travail. Avril 1987. Volume 8, Numéro 4.

23. Lachance, Jean-Pierre. Relevé du nombre d'employeur Réseau Saguenay Lac St-Jean. 1992-93. Direction de la planification stratégique . Emploi et Immigration Canada. Septembre 1992. 
du Canada de la région par l'intermédiaire des directeurs(trices) de ces bureaux.

- le processus a permis d'identifier huit (8) entreprises dont deux (2) ont été éliminées car l'équipement acquis ne répondait pas à celui identifié aux nouvelles technologies énumérées en annexe. Pour deux autres entreprises, les dirigeants n'ont pu être rejoint ou leur disponibilité ne permettait pas de les rencontrer.

- bref, les quatre entreprises ont fait partie de la sélection finale et les résultats de l'étude vous sont présentés plus loin.

- préalablement, pour obtenir l'information nécessaire, nous avons utilisé une méthode de recherche qualitative plus appropriée au type d'informations désirées. Les entrevues auprès de chaque entrepreneur ont été conduites dans un style d'animation semi-directif, c'est-à-dire que le déroulement de l'entrevue se faisait selon une séquence de questions fixées à l'avance à l'intérieur desquelles l'entrepreneur avait toute latitude possible pour y répondre. Les questions suivaient l'ordre du modèle développé par l'auteur. Ainsi, à partir d'un des éléments de l'infrastructure humaine, soit 
les activités de formation, nous nous intéressions dans un premier temps à la situation existante dans l'entreprise et ce, avant l'adoption du nouvel équipement. Par la suite, les interrogations portaient sur l'impact de l'achat de la machinerie. Nous poursuivions avec les contraintes dont les effets étaient jugés importants selon le modèle. Finalement, nous jetions un regard de l'impact sur d'autres éléments s'il y avait lieu. Le guide de l'entrevue figure à l'annexe III. Il faut également prendre en considération qu'il s'agit ici d'une étude de perceptions. La réalité qui y est présentée correspond à celle des chefs d'entreprises à un moment donné précis.

- une demande de recherche faite auprès de la commission de la Formation Professionnelle et d'Emploi Immigration Canada sur les contrats de formation octroyés à ces entreprises a permis de valider partiellement les données recueillies sur l'importance de la formation et sa diversification auprès du personnel.

- enfin, une rencontre s'est tenue avec Monsieur JeanClaude Dupéré, président de l'Association pour les enseignants en mécanique industrielle du Québec où nous avons échangé sur la problématique du secteur ainsi que sur 
l'exhaustivité de la liste des entreprises ayant acquis de nouvelles technologies au cours des dernières années. 


\section{CHAPITRE V \\ ETUUDS DE CAS}

Pour le profit des lecteurs, nous rapportons un résumé des discussions tenues avec chaque dirigeant d'entreprises. Chaque cas est présenté dans les pages qui suivent. L'analyse permettra également de faire ressortir les conclusions de l'examen de chaque variable.

\section{ENTREPRISE A}

L'entreprise A évolue dans le secteur de la fabrication des produits métalliques depuis plusieurs années. Avant d'entrer dans le vif du sujet, permettez-moi de faire une brève description de l'industrie qui facilitera aux lecteurs la compréhension du rôle stratégique de l'adoption de nouvelles technologies pour les entreprises du secteur.

L'emploi, au niveau du Québec, atteignit un sommet en 1981, avant de subir deux années de diminution suite à la récession qui a prévalu. En 1984, l'industrie commence à profiter de l'expansion économique qui prend son envol et les effectifs augmentèrent jusqu'en 1990, dépassant son sommet 
précédent. Par la suite, la mondialisation des marchés, la récession économique et l'absence de contrats d'investissement majeur dans la construction industrielle et commerciale firent que l'emploi chuta à un niveau plus bas encore que celui atteint en 1981-82. Devant ces conditions, les usines de ce secteur situées au Saguenay Lac st-Jean ont connu aussi leur part de difficultés et plusieurs fermetures, soit temporaires, soit définitives ont été enregistrées. Nous avons même constaté au cours de la dernière année, la disparition d'une firme dont l'équipement était à la fine pointe de la technologie.

L'entreprise A appartient à des gens du milieu ainsi qu'à des ex-travailleurs d'une usine de fabrication de produits métalliques qui déclara faillite. Dès le départ, compte tenu de l'actionnariat, il fût implanté un système de cogestion. Par la suite, l'entreprise, jusqu'à ce jour, n'a cessé de prendre de l'expansion au point que nous pourrions la classer dans les entreprises de taille moyenne.

L'entreprise a une production très diversifiée dont l'usinage, le soudage et l'assemblage de produits lourds et son équipement pour réaliser cette production est de type conventionnel. Selon le modèle conceptuel présenté dans les 
pages précédentes, il faut s'intéresser à cette étape de l'étude de cas à la situation qui prévalait dans l'usine avant l'adoption de nouvelles technologies.

Au chapitre de la direction, le directeur général est un homme considéré comme très dynamique et qui possède un baccalauréat en gènie. Selon lui, il est très important d'impliquer le syndicat et les employés dans la prise de décision. D'ailleurs, comme mentionné antérieurement, le style de gestion retenu est la formule de cogestion. La direction considère que le virage technologique s'inscrit commme une stratégie offensive qui vise à améliorer leur compétivité et la qualité de leurs produits. Ainsi, l'adoption de nouvelles technologies se présente pour l'entreprise comme la meilleure façon de demeurer dans le marché tenant compte que celle-ci oeuvre dans un marché fort concurrentiel dont la survie pourrait être menacé à plus ou moins longue échéance.

Quant à l'élément de l'infrastructure humaine retenu aux fins d'analyse, les activités de formation étaient considérées comme importantes aux yeux des dirigeants. Des besoins prévalaient avant même l'acquisition de l'équipement, mais ceux-ci étaient répondus à la pièce. Aucune 
planification structurée n'avait été mise en place par l'entreprise pour donner suite à la formation du personnel.

Suite à ce portrait de la situation qui prévalait dans l'entreprise avant l'achat de l'équipement, nous devons maintenant procéder à l'étape concernant le choix de l'équipement et l'impact de cette décision sur les emplois. Pour l'achat de l'équipement, le dirigeant mentionne que les superviseurs ont été consultés dans l'analyse et le choix de l'équipement retenu. De plus, la direction était consciente que des effets se répercuteront sur le personnel suite à l'achat. Ainsi, dans un premier temps, des rencontres avec le personnel avait été organisé afin de prévenir toute résistance au changement et fournir toute l'information pertinente. De plus, les propriétaires étaient également conscients que l'acquisition de l'équipement se traduirait par des besoins de formation auprès du personnel.

$\mathrm{Au}$ chapitre des impacts, le modèle prévoyait (4) contraintes dont les effets sont déterminants pour l'entreprise. La première contrainte se rapportait à la force du travail ou aux employés, en d'autres mots. Ainsi, quant à l'existence d'un intérêt pour les travailleurs à relever des défis, l'historique de l'entreprise et la 
participation des employés dans la propriété de l'usine venaient facilement répondre à cette interrogation. De plus, le degré de confiance des travailleurs envers la direction ne causait aucun problème compte tenu de la présence d'un syndicat et la formule de cogestion mis en place.

L'impact du nouvel équipement a eu comme conséquence l'observation de quelques soubresauts chez le personnel. Dans un premier temps, le dirigeant mentionna que les jeunes ont facilement accepter le changement. Plusieurs de ceux-ci avaient déjà au point de départ une formation qui leur avait permis de se familiariser avec la technologie. Chez les plus âgés, il a ressenti un sentiment d'insécurité chez certains, mais cette faible résistance n'a pas perduré. Les employés ont vite compris que la technologie leur permettrait de fabriquer un produit de meilleure qualité ce qui ne pourrait que se répercuter sur les ventes et sur leur sécurité d'emploi.

Au chapitre de la formation, celle-ci a été dispensée en premier lieu par les représentants-vendeurs de la machinerie. Par contre, les superviseurs se sont apperçus que l'utilisation efficiente de la machinerie exigerait une 
formation plus étendue. Une firme de l'extérieur sera engagée un peu plus tard pour répondre à ce besoin.

La seconde contrainte à prendre en considération dans le modèle de madame Majchrzak était celle de la planification du processus. Selon la personne rencontrée, cette variable n'a pas tellement eu d'effets dans l'adoption d'une nouvelle technologie. La situation économique qui prévalait a peutêtre eu une influence compte tenu du caractère rare des employés disponible, ainsi par conséquent aucune mobilité des travailleurs n'a èté observée. De même, les accords avec les fournisseurs de matière première n'ont pas eu besoin d'être modifiés. Seul élément à prendre en considération serait le rythme du processus. Selon le directeur général, l'entreprise doit s'attendre à ce que l'apprentissage de l'équipement se déroule sur une période assez longue. Au chapitre de la rentabilité, nos prévisions doivent s'établir à long terme.

La prochaine contrainte était reliée à l'évolution des marchés. Pour l'entreprise A, cette variable n'a pas eu d'effets significatifs suite à l'ajout d'équipements de haute technologie. Bien que vous améliorez la qualité du produit, 
l'impact est très minime sur la capacité à pénéter de nouveaux marchés selon les propos recueillis.

Enfin, la dernière contrainte était associée à la philosophie de gestion. Pour le dirigeant, l'entreprise A se donne comme mission d'être un des leaders de l'industrie en assurant un produit de qualité à sa clientèle et le bien-être de son personnel. Ainsi, toute la direction a pris une série d'engagements qui vont dans ce sens. La priorité est accordée aux ressources humaines et la prise de décision doit se faire au niveau le plus bas possible.

A savoir quelles seraient les conditions de succès pour réussir l'implantation d'une nouvelle technologie, le dirigeant a énuméré trois facteurs soit:

- il faut, avant même l'achat de l'équipement, être présent dans le marché.

- il faut apporter un soin au choix de l'outillage.

- il faut accorder un attention à la formation du personnel.

En conclusion, l'étude menée auprès de cette entreprise a conduit à adopter les variables énumérées par madame 
Majchrzak dans son modèle. Par ailleurs, il faut également prendre en considération certains éléments qui apparaissent importants dont:

- l'entreprise s'est définie une mission

- l'entreprise fait face à une concurrence très forte sur le marché et doit lutter pour maintenir sa survie

- l'adoption de nouvelles technologies s'inscrit dans une stratégie d'affaires

- l'entreprise est de taille moyenne

- l'entreprise a adopté des pratiques de gestion modernes

- la scolarité du dirigeant est de niveau universitaire

- l'entreprise accorde une priorité à la gestion des ressources humaines

ENTREPRISE B

L'entreprise $B$ fût fondée par la réunion de (5) cinq machinistes qui décidèrent de se lancer en affaires. Les ventes ont grimpé, mais vers la fin des années 80 , en l'espace de peu de temps, trois des propriétaires quittèrent 
pour partir chacun à leur compte une entreprise qui oeuvrera dans le même domaine.

Nous avons appliqué le même processus que la rencontre avec le dirigeant de l'entreprise A et avons questionné sur la situation qui prévalait avant l'achat du nouvel équipement. Selon les données recueillies, des problèmes au niveau du climat de travail semblaient prévaloir dans l'entreprise au point où le dirigeant mentionna qu'une firme d'études de l'extérieur est venue les conseiller sur le sujet. Son intervention a porté uniquement au niveau des cadres et des chefs de départements. Nous n'avons pu en savoir beaucoup plus sur cette question, sauf que le départ des trois actionnaires ne serait pas étranger au climat de travail qui prévalait.

$\mathrm{Au}$ chapitre de la formation du personnel, aucune planification à ce sujet n'a été élaborèe et aucune activité ne s'y est déroulèe, mais déjà des problèmes de qualité au niveau du produit fabriqué étaient observés et des plaintes des clients étaient formulées.

Nous nous sommes intéressés par la suite à la décision reliée à l'achat de l'équipement et son impact sur le 
personnel. Selon la personne rencontrée, cet achat s'est fait dans le milieu des années 80 rendant l'entreprise assez innovatrice comparativement à ses concurents. Le but visé était de sauver du temps et améliorer par la même occasion la crédibilité de l'usine. A savoir si les travailleurs ont été impliqués dans l'achat, le dirigeant a fait mention qu'aucune consultation n'a été faite; les employés ont tout simplement été mis devant un fait accompli. L'achat des machines était tout simplement relié à l'obtention de contrats par une grande entreprise.

Les impacts sur le personnel se traduisirent de diverses façons. Dans un premier temps, le dirigeant croyait que le fait d'obtenir de la machinerie à haute technologie permettrait moins d'erreur et procurerait une plus grande flexibilité. A cette époque, l'économie étant en expansion et la main-d'oeuvre qualifièe moins disponible, il se décida à identifier parmi le personnel ceux qui répondaient le mieux aux exigences pour devenir des opérateurs. Il confia le mandat de dispenser la formation à un ingénieur oeuvrant dans l'entreprise. Les résultats ne furent guère dans la direction prévue et les retours de pièces continuèrent d' augmenter. 
Le dirigeant constata également que le personnel semblait plus ou moins prendre au sérieux le rôle attendu. Selon la personne rencontrée, les travailleurs ont compris qu'ils pouvaient économiser du temps grâce à la rapiditè de la machinerie, mais la qualité laissait toujours à désirer. Les actionnaires mandatèrent alors un ingénieur pour se pencher sur le contrôle de la qualité et pour tenir des réunions avec le personnel sur le sujet.

Bref, l'entreprise a dispensé très peu de formation à son personnel si ce n'est celle fournie par les représentants-vendeurs lors de l'achat. Selon le dirigeant, aucune activité de formation provenant de l'extérieur de la firme n'est prévue, laissant aux ingénieurs la responsabilité pour ce faire.

Au chapitre des contraintes reliées à la planification du processus, aucun changement n'a été observé dans l'entreprise. Cette variable ne s'appliquerait pas selon la personne rencontrée. De plus, l'entreprise, qui n'étant pas syndiquée, verse des salaires plus élevés que la moyenne de I'industrie à ses employés, de sorte que le taux de roulement est faible. 
Concernant l'impact de la machinerie sur l'évolution des marchés, l'entrepreneur disait qu'il était important de modifier l'équipement conventionnel par de la nouvelle technologie. Par ailleurs, deux élément l'on empêché d'augmenter la vitesse de réalisation de cet objectif, soit la récession économique et le coût de la machinerie. Selon le dirigeant, avec la nouvelle technologie, il pourrait pénétrer plus facilement de nouveaux marchés.

Quant à la philosophie de gestion, le dirigeant a déclaré se situer plus du coté $x$ de la théorie de Mc Grégor bien qu'il a réalisé qu'il faudrait peut-être penser à susciter les employés à l'actionnariat de l'entreprise pour améliorer leur motivation. La décision à ce sujet n'est pas encore définitive. Pour la prise de décision, celle-ci revient aux dirigeants qui, quelque fois, auront soin d'en discuter avec les ingénieurs. Finalement, la priorité accordée aux ressources humaines pour les activités de formation n'est pas évidente.

A savoir quelles seraient les conditions de succès pour réussir l'implantation de nouvelles technologies, il mentionna deux facteurs soit: 
- le choix de l'équipement

- la participation du personnel

En conclusion, l'étude menée auprès de cette entreprise démontre que la direction a très peu impliqué les travailleurs dans la prise de décision et aucune planification sur l'infrastructure humaine n'a été mise en place. De plus, on observe un climat de travail et des attentes sur la qualité de la production qui ne semblent pas répondre aux attentes de la direction à ce sujet. Quant à l'élément de la formation du personnel, seul le minimum a été dispensé et cette variable ne semble pas être la priorité de la direction. Pour ce qui est de la gestion de l'entreprise - celle-ci est plutôt planifiée au jour le jour selon les propos du dirigeant.

Les éléments qui ressortent de cette étude de cas sont:

- l'entreprise applique une politique de gestion où les éléments de planification sont à peu près absents

- l'entreprise fait également face à une concurrence de plus en plus vive sur le marché

- l'entreprise est de petite taille 
- l'entreprise applique des méthodes de gestion qui ne cherchent pas à impliquer le personnel

- la scolarité des dirigeants est de niveau secondaire professionnel

- l'entreprise est non-syndiquée

- l'entreprise accorde très peu d'importance aux activités de formation; mais mise beaucoup sur l'aspect qualité

ENTREPRISE C

L'entreprise $\mathrm{C}$ a vu le jour suite à une décision de son propriétaire de se lancer en affaires. Comme l'usine précédente, celle-ci opère avec un nombre peu élevé de travailleurs permettant de la classifier parmi les petites entreprises. Elle se caractérise également, comme la précédente d'ailleurs, comme une sous-contractante de la grande entreprise.

En appliquant le modèle de madame Majchrzak, nous nous sommes intéressés dans un premier temps, à la situation qui 
prévalait dans l'atelier avant l'achat du nouvel équipement. Selon les dires de l'entrepreneur, l'usine est syndiquée et les relations avec l'autre partie sont plus ou moins bonnes. De plus, il a mentionné que l'entreprise n'a aucune culture de formation. Il appartient aux employés d'obtenir leur qualification au moment de l'embauche.

Par la suite, nous avons fait porter nos interrogations sur I'achat de l'équipement et l'impact sur le personnel. Concernant la décision de procéder au choix de l'équipement, l'entrepreneur a pris sur lui de visiter plusieurs manufacturiers et vérifier la qualité du produit. Il mentionna que cet exercice a exigé de sa part passablement de temps. Par contre, en aucun moment, il a eu recours à son personnel pour le choix de l'équipement. De même, aucune planification de l'impact de l'adoption d'une nouvelle machinerie n'a été réalisée. Comme il le mentionna, une partie de cette responsabilité est du ressort du fournisseur d'équipement qui devra assumer la formation du personnel.

Au chapitre de l'impact de la force du travail, le dirigeant à procédé à la désignation de deux travailleurs qui auront la responsabilité d'opérer la machine. Cette décision a été prise sans consultation auprès du syndicat. Une 
résistance au changement, s'est rapidement manifestée dans l'entreprise et ce, de plusieurs façons.

Premièrement, la formation a été dispensée uniquement aux opérateurs, le superviseur des opérations a été ignoré. Ainsi, un souffle de mécontentement est vite apparu dans l'entreprise. Le syndicat se plaint qu'il n'a pas été impliqué dans les décisions ce qui s'est traduit par des rencontres patronales-syndicales plus rigides qu'auparavant. Le climat de travail souffre d'un manque de motivation de la part de certains membres du personnel qui peuvent produire moins et avec une plus grande marge d'erreur que ceux qui opèrent la machine. Un sentiment d'insécurité s'est développé chez les travailleurs plus âgés. Bref, l'entreprise nage dans diverses complications depuis l'installation de cet équipement qui remonte à environ une douzaine de mois.

Par la suite, notre entrevue s'est dirigée sur les contraintes reliées à la planification du processus. Selon le propriétaire aucun changement n'a été observé tant sur le degré d'autonomie des différentes directions que les accords avec les fournisseurs. De même, aucun changement dans le nombre des travailleurs n'a été apporté. 
Quant aux contraintes reliées à l'évolution des marchés, cette variable n'est pas pertinente selon le proprietaire. L'acquisition de la machine est fait dans le but de demeurer concurrentiel et compétitif face aux autres ateliers et non en fonction d'aller chercher de nouveaux marchés.

Pour la philosophie de gestion, le dirigeant mentionna que le propriétaire a comme rôle de prendre des décisions alors que les travailleurs sont rémunérés pour produire. Par ailleurs, il est conscient qu'il fait face présentement à des difficultés reliées à une démotivation du personnel. D'ailleurs ce sentiment ou cette préoccupation se retrouve dans les écueils à éviter lors de l'implantation de nouvelles technologies. Ainsi, à ce dernier sujet, il mentionna:

- $\quad$ il faut impliquer les employés dès le départ

- le choix de l'équipement s'avère primordial

- il faut s'interroger sur les relations entre l'homme et la machine. La nouvelle technologie apporte ou implique des changements dans la gestion des entreprises.

En conclusion, parmi les éléments importants qui retiennent l'attention dans ce cas, mentionnons que: 
- l'entreprise fait face à une forte compétition sur les marchés

- l'Adoption de la nouvelle technologie s'inscrit dans une meilleure productivité

- l'entreprise est de petite taille

- la direction de l'entreprise repose sur l'hommeorchestre

- la scolarité du dirigeant est de niveau secondaire professionnel

- l'entreprise est syndiquée

- la direction accorde peu d'importance aux activités de formation, mais le contrôle de qualité est important

ENTREPRISE D

L'entreprise $D$ est une filiale d'un regroupement d'industries oeuvrant dans le secteur manufacturier et appartenant à un même propriétaire. Celle-ci a vu le jour au cours de la dernière période d'expansion économique et se distingue par un haut degré de technicité comparativement aux autres du même groupe. 
L'atelier oeuvre dans la fabrication de diverses pièces d'équipement et sa direction est assumée par un ingénieur de formation. Parmi les valeurs de ce dirigeant, l'implication du personnel s'avère important dans un processus de réussite. De plus, l'entreprise de donne comme mission de produire un produit de qualité et de devenir un des principaux leaders sur le marché.

En suivant les mêmes étapes que les études précédentes, nous avons interrogé le dirigeant sur la situation qui prévalait avant l'achat de l'équipement. Comme l'entreprise est récente, une des premières actions effectuées fût de dispenser une formation de base d'une durée d'environ neuf (9) mois à tout le personnel qui venait d'être embauché. Ainsi, l'entreprise pouvait faire connaitre ses valeurs et ses attentes à son personnel et disposer d'une main-d'oeuvre compétente.

Comme l'entreprise était récente, on voulait également par souci d'efficacité compter sur de la machinerie à la fine pointe de la technologie. Pour procéder au choix, l'entreprise a mis sur pied un comité formé de cadres et d'ingénieurs en mécanique pour étudier les différentes possibilités. De plus, la direction était consciente que 
l'achat d'équipement se répercutera sur le personnel de sorte que des moyens ont été mis en place pour que les travailleurs soient informés des démarches. Il faut également souligner que l'entreprise est syndiquée et qu'une clause dans la convention stipule que les employés doivent être avisés deux mois à l'avance de tout changement technologique.

Concernant les effets de l'achat sur le personnel, la direction avait planifié le choix des opérateurs qui se sont vus octroyer une formation sur la nouvelle machinerie par le fournisseur de l'équipement. Pour les autres, la politique d'informations, la présence du syndicat, la volonté du personnel à relever des défis, sont autant d'éléments qui ont contribué à un environnement où le travail d'équipe prévaut. Bref, aucune résistance n'a été perçue. De plus, selon le dirigeant, étant en période de croissance et aucun n'emploi n'étant menacé, ces facteurs ont exercé un effet sur l'esprit d'équipe et sur le degré de motivation du personnel. De même, la motivation se répercutera sur la qualité du produit, ainsi tous en ressortent gagnants.

Nos questions se sont dirigées par la suite sur la planification du processus. Cette variable ne jouerait pas un rôle important sur l'adoption de la nouvelle technologie 
selon notre interlocuteur. Seul élément à considérer, serait le rythme du processus. Une entreprise qui prend le virage technologique doit pouvoir compter sur un certain délai avant d'en avoir une parfaite maftrise et penser rentabilité.

Au chapitre de la contrainte reliée aux prévisions du marché, le dirigeant n'établit aucun lien entre l'achat de machinerie et l'obtention de nouvelles commandes. Selon sa perception, l'entreprise doit déjà avoir des commandes en carnet et être présent sur le marché avant de recourir à la machinerie. Toute action inverse pourrait conduire à un échec.

Enfin, selon le modèle développé, la philosophie de gestion est la dernière contrainte à prendre en considération. Dans le cas de l'entreprise D, la politique de gestion se résume à obtenir la participation du personnel dans la prise de décisions. Les relations avec la partie syndicale se déroulent rondement dans un respect mutuel selon les dires du dirigeant.

Quant au choix des conditions de succès il énuméra les suivantes: 
- il faut être présent sur le marché avant de recourir aux nouvelles technologies

- le choix de l'équipement est très important

- l'entreprise doit compter sur une main-d'oeuvre qualifiée

En conclusion, la situation vécue dans l'entreprise épouse relativement bien le modèle présenté dans ce mémoire. Seul le degré d'importance des divers éléments semble varier en particulier les contraintes reliées au processus et aux prévisions du marché. Par ailleurs, il faut prendre en considération les éléments suivants:

- l'entreprise s'est définie une mission

- l'entreprise est de taille moyenne

- l'entreprise a adopté des pratiques de gestion modernes

- la scolarité du dirigeant est de niveau universitaire 
- les dirigeants accordent une importance aux qualifications de la main-d'oeuvre 


\section{CHAPITRE VI \\ ANALYSE DES RÉSULTATS}

Dans un premier temps, nous allons nous attarder aux taux de diffusion de la nouvelle technologie dans les ateliers d'usinage au Saguenay Lac st-Jean. Ce concept prend son importance vu que les experts s'accordent pour reconnaitre que les entreprises n'ont d'autres choix que de recourir à la technologie pour améliorer leur position concurrentielle. Ainsi six (6) entreprises sur un total de soixante-dix huit (78) ont procédé à l'achat de nouveaux équipements soit moins que $8 \%$. Ce résultat pourrait s'avérer inquiétant sachant que le taux de diffusion dans la grande entreprise tend à s'accrô̂tre et que les petites entreprises opèrent bien souvent comme sous-traitants des premières. D'ailleurs, au cours des mois qui suivirent nos entrevues, un atelier d'usinage avisait ses travailleurs que l'entreprise fermerait ses portes pour une période indéfinie. Cet arrêt découle $d u$ contexte économique difficile. Le dirigeant mentionnait qu'il ne fermerait pas ses portes s'il parvenait à décrocher des contrats demain matin. De même, ce porteparole disait estimer que la région du Saguenay Lac st-Jean 
compte présentement trop d'usines de fabrication d'équipements industriels.

Cette situation fait ressortir deux éléments primordiaux. Dans un premier temps, la compétition est féroce dans ce domaine et seul les petites et moyennes entreprises qui sauront combler les attentes des plus grandes qui fournissent les contrats, connaîtront du succès et survivront. Deuxièmement, la grande entreprise fait face à la mondialisation des marchés et l'augmentation de la concurrence qui en découle. Parmi les moyens pour améliorer sa productivité et diminuer les coûts de production, cellesci n'hésite pas à investir dans des dépenses reliées à l'équipement et la machinerie, dans la formation du personnel ainsi que les normes de qualité.

En retour, la grande entreprise s'attend à ce que les entreprises qui sous-contractent puissent répondre aux mêmes normes de qualité et ce, bien souvent, à un coût moindre. Il n'est donc pas surprenant que dans toutes les entreprises rencontrées, les dirigeants ont mentionné que le but visé par I'acquisition d'équipements reliés aux nouvelles technologies était soit d'augmenter la productivité soit d'améliorer la 
qualité. Ces deux objectifs sont bel et bien en étroite relation avec la problématique décrite au départ.

Par la suite, nous nous sommes intéressés à la situation qui prévalait dans les entreprises avant l'achat de l'équipement, et ce, en particulier pour l'élément de l'infrastructure humaine retenu, soit les activités de formation. Selon les études de cas, il en ressort que seulement deux entreprises avaient mis en place une politique des ressources humaines qui accordait une priorité à cette fonction. De plus, ces deux usine ont différents points en commun soit:

- la direction est assumée par le dirigeant qui possède une formation de niveau universitaire

- le nombre d'employés permet de classifier les deux usines comme des entreprises de taille moyenne

- les deux entreprises subissent une forte compétition qui pourraient déterminer leur survie

- les directions de ces usines ont des pratiques de gestion plus modernes 
- les deux entreprises mettent l'accent sur la qualité du produit et du service

Pour les deux autres entreprises sous étude, aucune activité de formation prévalait à l'intérieur de leur murs préalablement à l'adoption de nouvelles technologies. De même, des points communs ressortent soit:

- les propriétaires ou dirigeants ont créé leur propre entreprise

- les deux entreprises peuvent être classifiées comme des petites organisations compte tenu de leurs effectifs en emploi

- les deux organisations sont sous-contractantes de plus grandes entreprises et leur style de direction repose sur le propriétaire. Les employés sont peu ou pas impliqués dans la prise de décisions.

Bref, sans pouvoir généraliser, la taille des organisations, la scolarité du dirigeant, le style de gestion sont autant de facteurs qui peuvent avoir une incidence sur le recours à des activités de formation. D'ailleurs des 
études ont démontré une relation entre l'importance de la formation et la taille des organisations.

Au chapitre du choix de l'équipement, tous reconnaissent l'importance de cet élément dans le succès relié à l'adoption de nouvelles technologies. Ce résultat s'inscrit également dans le même sens que le modèle de madame Majchrzak qui retenait cet élément comme un des plus prioritaires.

Concernant les effets de premier ordre, soit l'impact du choix de l'équipement sur les opérateurs de la machinerie, tous ont procédé par une formation distribuée par le fournisseur de l'équipement. Seulement une entreprise demandera une expertise d'une firme de l'extérieur pour pouvoir compter sur une utilisation plus efficiente de la machinerie. Par ailleurs, il faut souligner que les activités de formation ont été étendues à d'autres catégories de personnel chez des entreprises de taille moyenne. Pour les entreprises de petite taille, nous n'avons pas perçu que la formation pourrait s'avérer une activité importante à leurs yeux. Il faudra peut-être vérifier dans une autre étude si les entreprises qui adoptent de nouvelles technologies en fournissant au personnel de meilleures qualifications ont de meilleurs résultats. Il demeure que 
les recherches démontrent que l'importance de l'information progresse avec la taille des entreprises et cette affirmation se confirme selon les études de cas.

Parmi les autres effets de l'adoption de nouvelles technologies, l'auteur mentionnait qu'il fallait apporter une attention spéciale à la résistance au changement de la part du personnel. A ce sujet, deux dirigeants disent éprouver des difficultés avec le personnel passant de la résistance de la part de certains travailleurs à des confrontations lors de rencontres patronales-syndicales. Ce phénomène s'explique par le sentiment de peur et de méfiance qui peut s'installer chez les employés suite à l'approche de changements technologiques. Cette méfiance n'est pas étrangère et indépendante du climat qui prévaut dans l'entreprise. Si de bonnes communications sont maintenues entre la direction et le personnel de l'entreprise, cela contribue à tempérer le climat de crainte ou d'incertitude. Pour les deux entreprises ayant vécu un tel problème, les dirigeants ont justement mentionné ne pas avoir impliqué les employés dans la décision d'acquérir de nouveaux équipements.

Dans le cas d'une entreprise, avant même l'achat du nouvel équipement, une firme avait été engagée pour mener une 
étude sur le climat de travail dans l'entreprise et pour apporter des recommandations pour l'améliorer. Lors de la rencontre, le dirigeant rapportait justement la perte de trois contremaitres au cours des dernières années qui sont devenus des compétiteurs de son entreprise et des problèmes de motivation du personnel.

Pour l'autre, le dirigeant mentionnait rencontrer de la résistance de la part des travailleurs, en particulier des travailleurs plus âgés. De même, il a observé une certaine démotivation de la part de certains machinistes oeuvrant sur des machines traditionnelles face aux machinistes profitant de la nouvelle technologie.

Pour les deux autres entreprises racontant ne pas avoir observé d'impact, pour l'une, le dirigeant faisait part que la tenue à jour des informations auprès du personnel sur le sujet, le sort de l'entreprise qui repose sur l'augmentation de la productivité et des rencontres fréquentes entre les employés et les superviseurs ont permis d'éviter bien des complications. Autre facteur qui a joué un certain rôle est la présence d'une main-d'oeuvre relativement jeune. Par contre, certains travailleurs plus âgés ont démontré des 
signes d'inquiétude mais la direction a su prendre action rapidement.

Le dirigeant de notre dernière entreprise disait ègalement ne pas avoir encore rencontré de résistance. Selon lui, l'ouverture récente de l'atelier, le climat économique qui prévaut, de bonnes communications avec la partie syndicale sont tous des éléments ayant favorisé un climat de travail excellent. De plus, le fait de rester competitif et de produire des pièces de qualité a eu un effet d'entraînement supplémentaire sur les travailleurs.

Bref, au chapitre de la résistance du personnel, les deux entreprises qui ont éprouvé des difficultés à ce sujet ont des points en commun. Ces deux entrepreneurs dirigent des entreprises non syndiquées, de petite taille et appliquent un style de gestion où la consultation du personnel est peu sollicité. Par ailleurs, ces deux mêmes individus soulignaient parmi les conditions de succès de l'adoption de nouvelles technologies le recours à la participation du personnel et leur implication dès le départ. Cette apparente contradiction fait ressortir un des problèmes souvent mentionnés lors de nos consultations avec des petites et moyennes entreprises soit des besoins de formation en 
gestion, dont en ressources humaines surtout chez les dirigeants des petites organisations.

Concernant la variable ou contrainte se rapportant à l'évolution des marchés, tous les directeurs rencontrés ont été unanimes à souligner que l'entreprise doit déjà oeuvrer dans un marché bien défini avant de faire l'acquisition de nouvelles technologies. Ainsi, leurs propos s'écartent du modèle conceptuel qui mentionnait que cette contrainte pourrait exercer un certain rôle ou avoir une certaine influence. Selon eux, les petites et moyennes entreprises ne peuvent se permettre d'automatiser la production en vue d'acquérir de nouveaux marchés car le coût de l'équipement est trop élevé et sa rentabilité ne peut se concevoir qu'à long terme. Par ailleurs, bien souvent le recours à de nouvelles technologies est imposé en partie par l'obtention de contrats additionnels de la grande entreprise qui exige tellement de normes de qualité et de précision qu'un jour ou l'autre l'entreprise n'a plus guère de choix.

Par rapport à la planification du processus, les personnes rencontrées ont rapporté aucun impact significatif en relation avec l'achat de l'équipement. Selon eux, aucun changement n'est survenu dans le degré d'autonomie des 
différentes direction sur le processus. De même, les accords avec le fournisseur n'ont pas été modifiés. Seul élément important est la volonté exprimée de faire adopter par le personnel les mérites d'une politique de qualité totale. Ainsi, à leurs yeux, les efforts doivent être dirigés vers l'accréditation des normes de qualité et leur maintien. Pour certains dirigeants, cette valeur vient par ordre d'importance bien avant la formation du personnel. On ne semble pas faire de relation entre les principes de qualité totale et une formation adéquate du personnel. De plus, certains ont mentionné avoir eu recours plutôt à l'embauche d'une main-d'oeuvre qualifiée qu'à la formation d'une partie des ressources humaines à l'emploi de l'entreprise.

Finalement pour la dernière contrainte sous étude, soit la philosophie de la gestion, deux entrepreneurs disaient croire à l'importance de la motivation et à l'implication du personnel dans la prise de décisions. Pour les deux autres, le style de gestion prévalant dans l'entreprise se rapprochait plus de l'homme orchestre où le dirigeant est seul à tout faire et à prendre les décisions. Ainsi, il ne faut pas se surprendre que la priorité accordée aux ressources humaines soit le lot des deux dirigeants disant impliquer le personnel. Autre rapprochement qu'il faut 
signaler, les deux gestionnaires ci-haut mentionné, ont la destinée d'entreprises de plus grande taille que les deux autres.

Enfin, aux yeux des dirigeants rencontrés, les conditions de succès suite à l'adoption de nouvelles technologies sont nombreuses. La sélection de l'équipement est le critère le plus prioritaire et le plus souvent mentionné. Par la suite, la présence dans le marché et l'obtention de contrats au préalable, la participation et l'implication du personnel sont les autres conditions énumérées. Une seule entreprise fera de la formation du personnel une condition de réussite. 
CONCLUSION

Nous voulions vérifier dans le cadre de ce mémoire si les entreprises qui adoptaient de nouvelles technologies surtout les petites et les moyennes tenait compte de l'impact sur les divers éléments de l'infrastructure humaine dont, en particulier, les activités de formation. Selon les études de cas, les résultats varient selon la taille de l'entreprise, la scolarité du dirigeant, le style de gestion, la présence d'un syndicat, etc. Ainsi, nous constatons que les entreprises qui ont eu recours à de nouvelles technologies ont certain point en commun dont de subir une forte compétition, de dépendre des grandes entreprises pour une bonne partie de leurs chiffres d'affaires et de recourir à de nouvelles machineries dans le but d'améliorer leur productivité ou de survivre.

Par ailleurs dans le modèle conceptuel présenté, il était prévu que des problèmes de gestion ou de direction pourraient survenir si la stratégie d'adoption de nouveaux équipements n'est pas planifiée ou assumée. A ce chapitre, les résultats démontrent le bien-fondé de cette assertion, sauf que nous pouvons différencier les entreprises qui ont eu 
recours à une planification contrairement aux autres. Ainsi, nous retrouvons les caractéristiques suivantes :

- les entreprises sont de taille moyenne

- les dirigeants ont un niveau de scolarité plus élevé

- les entreprises sont syndiquées

- l'entreprise applique des pratiques de gestion plus ouvertes et modernes.

Ces mêmes entreprises ont mis sur pied des activités de formation pour l'ensemble du personnel et accordent une certaine importance à celles-ci.

Du coté des petites entreprises, celles-ci ont également procédé à l'achat de machinerie provenant de l'étranger, mais se sont aussi arrêtées à l'investissement monétaire. Ainsi, des difficultés ou des résistances parmi le personnel ont vu le jour. Les activités de formation se sont limitées à celles fournies par les représentants-vendeurs et s'adressaient uniquement aux opérateurs. Pourtant les 
dirigeants de ces mêmes entreprises sont conscients des problèmes rencontrés et des solutions sont mentionnées comme des conditions de succès pour l'adoption de nouvelles technologies. Par ailleurs, les dirigeants des petites organisations sont souvent appelés à jouer plusieurs rôles et leur caractère entrepreneurial ne permet pas toujours de compenser des lacunes en gestion, si souvent constatées.

Bref, compte tenu du nombre limité d'entreprises rencontrées, nous ne pouvons généraliser sur ce sujet, mais nous percevons que l'impact des changements technologiques sur les éléments de l'infrastructure humaine se répercutera différemment sur les entreprises selon la présence de diverses variables. Nous constatons également que les activités de formation prennent de plus en plus d'importance selon les mêmes variables.

Nous pourrions donc émettre l'hypothèse que sur une grande échelle, nous pourrions trouver une corrélation significative entre ces variables et le recours à la formation du personnel dans les PME.

Il serait intéressant de vérifier si les entreprises qui recourent le plus à la formation du personnel obtiennent une 
utilisation plus efficiente des possibilités de la nouvelle machinerie et par conséquent, de meilleurs résultats financiers. Nous laissons cette piste de recherche à des futurs travaux dans ce domaine qui demeure encore bien peu exploité. 


\section{BIBLIOGRAPHIE}

BELAND, Pierre et al., La formation professionnelle: les défis des années 1990., Revue le marchè du travail, mars 1992.

BELANGER et al., Gestion stratégique des ressources humaines, Gaetan Morin éditeur, $662 \mathrm{p}$.

BENOIT, Carmelle et ROUSSEAU, Marie-Diane, La gestion des ressources humaines dans les PME au Québec, Gouvernement du Québec, MMSRFP, Direction de la recherche, mai 1992, $336 \mathrm{p}$.

BORDELEAU, Yvan et al., Comprendre l'organisation: approches de recherche, Éditions Agence D'Arc Inc., Montréal, 1983, $198 \mathrm{p}$.

CANADA, Conseil économique du Canada, Le recentrage technologique, innovations, emplois,adaptations, 1987, ottawa, $43 \mathrm{p}$. 
CANADA, Emploi Immigration Canada, Le nouveau mode d'emploi: profil de la croissance du marché du travail, 1989, 32 p.

CANADA, Statistique Canada, Enquête sur la formation et le développement des ressources humaines, 1989

GAGNON, Yves-Chantal et LANDRY, Maurice, La planification des changements technologiques et la survie des PME: réflexion sur un cas vécu, Revue PMO, volume 5, no. 2 .

JULIEN, Pierre-Andre et al., La diffusion des nouvelles technologies dans trois secteurs industriels, GREPME, Université du Québec à Trois-Rivières, conseil de la science et de la technologie, avril 1988, 46 p.

LACHANCE, Jean-Pierre, Relevé du nombre d'employeurs, Gouvernement du Canada, EIC, Direction de la planification stratégique, sept. 1992, 24 p.

LAROUCHE, Gabriel, L'impact des machines-outils à commandes numériques sur l'emploi et les ressources humaines de l'entreprise, Revue le marché du travail, avril 1987, volume 8 , no. 4 . 
LES AFFAIRES, La formation sur mesure: une formule adaptée aux besoins des PME, 8 août 1992, cahier spécial

MAJCHRZAK, Ann, The human side of factory automation, The Jossey-Bass Management Series, 1988, 390 p.

MAHE DE BOISLANDELLE, Henri, Gestion des ressources humaines dans les PME, Edition Economica, 1988, $322 \mathrm{p}$

NYAHOHO, Emmanuel, Impact des changements technologiques dans les secteurs d'activité économique du Québec et évolution structurelle de l'emploi, Revue le marché du travail, volume 7 , no. 5, mai 1986, 192 p.

QUÉBEC, Ministère du travail, Les changements de l'organisation économique, Revue le marché du travail, analyse du mois, août 1992

WRIGHT, Ruth, strategic connexions: technology, innovation and labour relations, A Conference Board of Canada Report Compensation Research Centre, march 1991, 34 p. 


\begin{abstract}
ANNEXE I
DESCRIPTION DES NOUVELLES TECHNOLOGIES

SELON LE NIVEAU DE COMPLEXITÉ
\end{abstract}

SECTEUR PRIMAIRE ET SECONDAIRE

Pour les secteurs primaires et secondaires, on peut distinguer les techniques d'équipements des technologies de systèmes intégrés de production et des technologies des nouvelles matières.

TECHNOLOGIES D'ÉQUIPEMENTS

1.Machines et outils modernes. Équipements de type traditionnel, mais plus performants, par exemple les machines verticales dans l'industrie des pâtes et papiers.

2.machines à commandes ou à contrôles numériques(MOCN). systèmes de contrôle informatisé (avec instructions préenregistrées et codées sur papier et sur bande magnétique) adjoints aux équipements anciens ou modernes, et servant à 
mieux diriger leur fonctionnement et à donner toutes les informations nécessaires à leur utilisation et à leur entretien.

\section{Véhicules filoguidés, système d'alimentation automatique,} d'emballage moderne, de stockage automatique, etc. Equipements servant à accélérer les approvisionnements des machines, à faciliter le passage des matières ou des produits semi-finis entre eux, à accélérer l'emballage au besoin et à améliorer le stockage une fois la production terminée. Ces machines peuvent être plus rapides jusqu'à automatiques grâce à l'informatique. Par exemple, elles peuvent permettre d'identifier automatiquement les pièces et les produits à l'aide de robots.

4.Robots. Appareils automatiques programmables, susceptibles d'effectuer des tâches de manipulation grâce à un ou plusieurs bras mécaniques articulés. Ces bras peuvent être contrôlés par un opérateur, fonctionnant alors suivant une séquence préétablie, ou être reliés à une commande numérique.

\section{Technologie de regroupement de pièces ou de matières.} organisation de pièces ou d'éléments de production par groupe ou par famille dans le but de faciliter des opérations 
standardisées de fabrication et de permettre aux machines de les "reconnaitre" plus facilement.

\begin{abstract}
6.Conception assistée par ordinateur (CAO) ou dessin assisté par ordinateur (DAO). Logiciels de divers types ayant pour but de préparer la production à l'aide d'un ordinateur permettant de modéliser (au besoins à trois dimensions) les plans de production (les pièces, les assemblages, etc.), de les analyser (poids, volume, centre de gravité, etc.), de simuler les mouvements, de réaliser les dessins automatiquement et de stoker les données et leurs paramètres. La CAO-DAO permet à l'ingénieur ou au dessinateur de réduire le nombre de calculs, de diminuer le temps d'étude des devis et de faciliter leur modification ou leur correction.
\end{abstract}

7.Fabrication assistée par ordinateur (FAO). Production à l'aide de machines programmables, telles que les machines à contrôles et à commandes numériques, mais surtout à l'aide de robots ou de manipulateurs automatiques programmables, soit à structures de commandes relativement simples ou répétitives, soit polyvalentes et adaptables (avec plusieurs degrés de liberté, actuellement jusqu'à six). Elle peut comprendre aussi la manutention automatisée et la technologie de groupe. Les robots peuvent charger et décharger les machines, 
manipuler des outils, usiner, monter, souder, réaliser des traitements thermiques, des opérations de peinture et de finition, etc.

8.CFAO ou centre d'usinage. Intégration de la cAO et de la FAO à partir d'une base de données communes. Ces centre d'usinage peuvent être très complexes; ils permettent de relier toutes les opérations et de les gérer selon une structure globale de production; c'est ce que les Américains appellent un "protocole de systèmes de fabrication" (manufacturing automation protocol) et les Européens un "système architectural ouvert".

9.Ateliers flexibles. Centres d'usinage affectant une partie de la production, tout en la rendant très flexible grâce à des robots tâches multiples, ou la production au complet, selon une production du genre"système intégré de production par ordinateur", (integrated flexible manufacturing system ou FMS).

10.Gestion de la production assistée par ordinateur ou "productique". Systèmes informatisés intégrés pouvant comprendre toute la gestion, comme on le verra plus loin. Ils peuvent englober l'entretien assisté par ordinateur 
(EAO), le contrôle de la qualité assisté par ordinateur (CQAO), etc. dans le but de mieux contrôler le cycle de production et de diminuer tous les délais en rendant la production flexible et les délais le plus courts possible (dans le genre du système japonais ou à "délais zéro"), etc.ce domaine qui demeure encore bien peu exploité. 
ANNEXE II

ÉTAPES A SUIVRE POUR L'IDENTIFICATION DES IMPACTS RELIÉS AUX RESSQURCES HUMAINES SUITE A I'ADOPTION DE NOUVELLES TECHNOLOGIES DE FABRICATION

\begin{tabular}{|c|c|c|c|c|}
\hline \multirow[t]{2}{*}{ Gontraintes } & \multirow[t]{2}{*}{ Equipements } & \multicolumn{3}{|c|}{ Effets } \\
\hline & & 1 & II & T T T \\
\hline
\end{tabular}

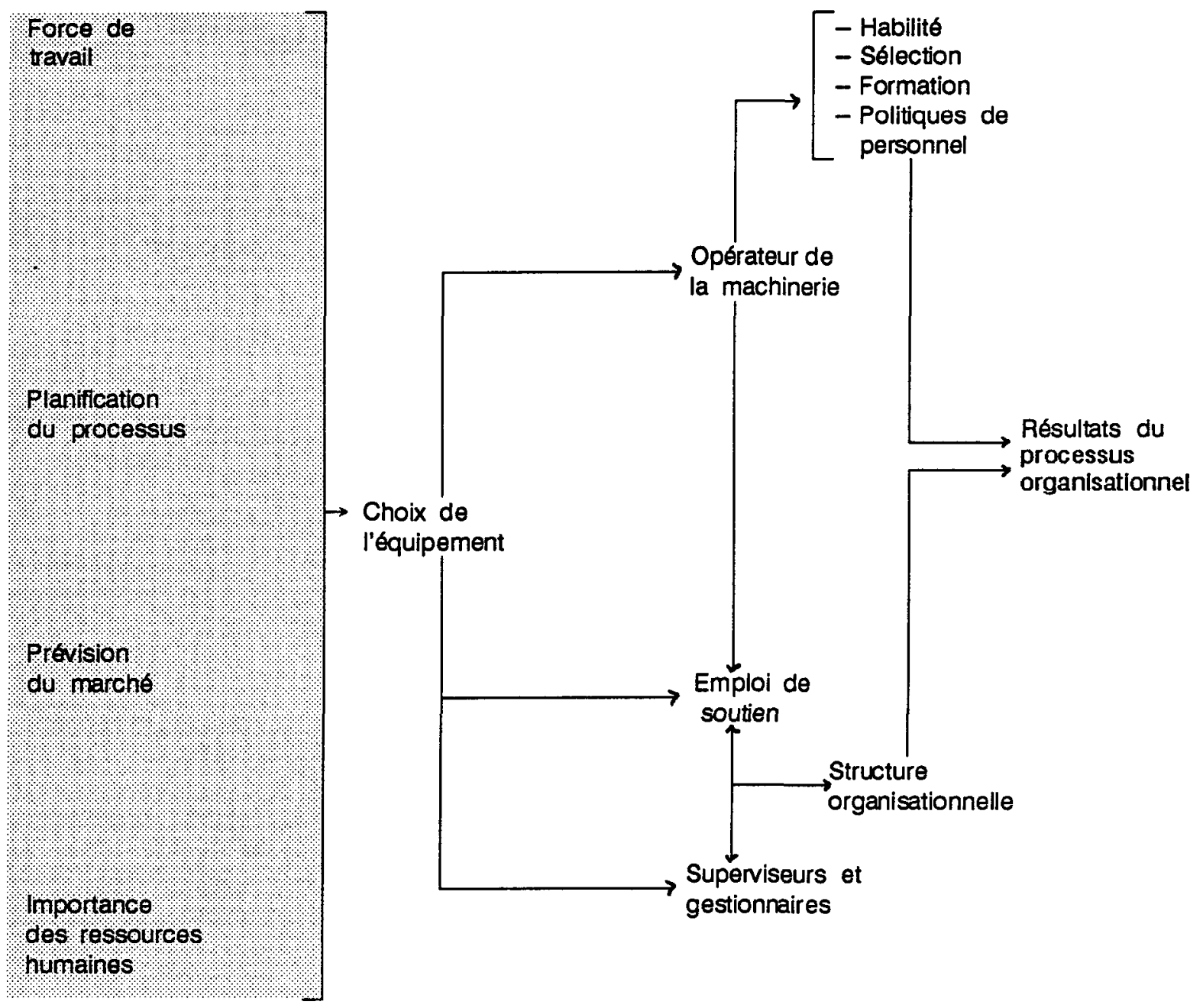

PROCESSUS DE CHANGEMENT

24 Tiré de Majchrzak, Ann. op.cit. Traduction libre 
ANNEXE III

GUIDE D' ENTREVUE

NOM DE L'ENTREPRISE:

NOM DE LA PERSONNE RENCONTRÉE:

DATE DE LA RENCONTRE:

NOMBRE D'EMPLOYÉS: SYNDICAT :

PARTIE -I-

(Situation existante avant le nouvel équipement)

Est-ce que vous dispensiez de la formation au personnel? Si oui, le type et la durée.

La date de l'adoption du nouvel équipement

Type d'équipement.

But de l'adoption du nouvel équipement. 
PARTIE -II

\section{(IMPACT)}

Avant l'achat, aviez-vous planifié l'impact des nouveaux équipements sur le personnel et les besoins de formation Si oui, quelles actions ont été entreprises?

PARTIE -III-

\section{(Impacts sur les contraintes selon l'importance)}

\section{A. EMPLOYÉS}

Avant existait-il un intérêt pour le travailleur à relever des défis?

Quel était le degré de confiance des travailleurs envers la direction?

Quelle type de main-d'oeuvre aviez-vous? Qualifié?

Non qualifié?

Quel a été l'impact du nouvel équipement sur la maind'oeuvre?

Plus motivé?

Plus de responsabilité?

Qui assure la formation?

Les superviseurs?

Quel a été le rythme du processus?

B.PIANIFICATION DU PROCESSUS

Degré d'autonomie des gestionnaires? 
Accords avec les fournisseurs?

\section{MARCHE}

Capacité à pénétrer de nouveaux marchés?

Non atteinte des prévisions?

Croissance de vente? Planification à long terme peut amener et demander de la flexibilité?

D. GESTION

Système de motivation du personnel:

Prise de décision:

Priorité accordée aux ressources humaines:

E. AUTRES

\section{Conclusion}

Si nous voudrions déterminer les conditions de succès pour l'implantation de nouvel équipement, sachant que 50\% des entreprises ne réussissent pas, que mentionneriez-vous?

Écueils? 